TRANSACTIONS OF THE

AMERICAN MATHEMATICAL SOCIETY

Volume 360, Number 11, November 2008, Pages 6065-6087

S 0002-9947(08)04451-6

Article electronically published on June 16, 2008

\title{
RANDOM WALKS ON PERIODIC GRAPHS
}

\author{
TAKAHIRO KAZAMI AND KÔHEI UCHIYAMA
}

\begin{abstract}
This paper concerns random walks on periodic graphs embedded in the $d$-dimensional Euclidian space $\mathbf{R}^{d}$ and obtains asymptotic expansions of the Green functions of them up to the second order term, which, expressed fairly explicitly, are easily computable for many examples. The result is used to derive an asymptotic form of the hitting distribution of a hyperplane of codimension one, which involves not only the first but also second order terms of the expansion of the Green function. We also give similar expansions of the transition probabilities of the walks.
\end{abstract}

\section{INTRODUCTION AND RESULTS}

We study in this paper a class of random walks on periodic graphs embedded in the $d$-dimensional Euclidian space $\mathbf{R}^{d}$. A random walk of the class may be regarded as a Markov additive process and we adapt the method devised for, or apply the results obtained for, Markov additive processes (e.g. 1], 3], [5], 4, 12]) to compute asymptotic expansions of the Green function of the walk. The result obtained here is used to compute an asymptotic form of the harmonic measure of the random walk on a half space in the case when a reflection principle is available. The identification of its principal term involves not only the first but also second terms of the expansion of the Green function. We give fairly simple expressions to the characteristics appearing in the expansion, of which we shall compute explicit forms for many examples. To facilitate the computation we advance useful consequences resulting from several kinds of symmetry of the walks. Some of the examples (especially Example 6) exhibit interesting features of the walks. We also include a local central limit theorem, viz., an expansion of the transition probability.

The periodic graph (or lattice) $(V, E)$ considered in this paper is an infinite graph with the vertex set $V \subset \mathbf{R}^{d}$ and the periodic structure given by a set of periods $e_{1}, \ldots, e_{d}$ which are linearly independent vectors of $\mathbf{R}^{d}$. Any edge $e=(u, v) \in E$ is an ordered pair of two vertices $u, v \in V$ (the case $u=v$ is permitted). The periodicity is given by means of an additive group

$$
\Gamma=\left\{\gamma_{x}: x \in \mathbf{Z}^{d}\right\}, \quad \gamma_{x}:=x_{1} e_{1}+\cdots+x_{d} e_{d},
$$

where $x=\left(x_{1}, \ldots, x_{d}\right)$ : each $\gamma \in \Gamma$ acts on the vertex set $V$ by $v \mapsto \gamma+v$ and if $(u, v) \in E$, then $(u+\gamma, v+\gamma) \in E$. In this circumstance we can choose a subset $T \subset V$, called a fundamental set, such that the classes $\xi+\Gamma$, as $\xi$ ranges over $T$, constitute a partition of $V$, or equivalently, the set of its shifts $\gamma+T, \gamma \in \Gamma$, makes

Received by the editors July 26, 2006 and, in revised form, November 21, 2006.

2000 Mathematics Subject Classification. Primary 60G50; Secondary 60J45.

Key words and phrases. Asymptotic expansion, Markov additive process, periodic graph, Green function, hitting distribution of a line.

(C)2008 American Mathematical Society 6065

Reverts to public domain 28 years from publication 
a partition of $V$. This induces a natural projection $\pi_{T}$ of $V$ onto $T: \pi_{T}(u)=\xi$ if $u-\xi \in \Gamma$. Conversely, $T$ may be regarded as a set of representatives of equivalence classes determined by $\pi_{T}$. (If $T\left(\subset \mathbf{R}^{d}\right.$ ) and $\Gamma$ are given in advance, the vertex set $V$ may in general be defined as a direct sum of $\gamma+T$ over $\gamma \in \Gamma$, which are not necessarily disjoint as point sets of $\mathbf{R}^{d}$ (hence on a point of $\mathbf{R}^{d}$ multiple vertices may sit); for simplicity, and in order to be consistent with the description given first, we suppose in this paper that $\gamma+T$ are disjoint for different $\gamma$ 's.)

The random walk $X_{n}$ on a periodic graph $(V, E)$ is a Markov process on $V$ whose transition law,

$$
p_{E}(u, v)=P\left[X_{n}=v \mid X_{n-1}=u\right]
$$

is such that $p_{E}(u, v)>0$ for $(u, v) \in E, p_{E}$ is periodic (i.e., $p_{E}(u+\gamma, v+\gamma)=$ $p_{E}(u, v)$ for $\left.\gamma \in \Gamma\right)$, for each vertex $u \in V, \sum_{v:(u, v) \in E} p_{E}(u, v)=1$ and $p_{E}(u, v)=0$ for $(u, v) \notin E$. It is supposed irreducible, namely from any vertex every other vertex can be reached by a path made up of vertices $u_{1}, \ldots, u_{n}$ such that $\left(u_{k}, u_{k+1}\right) \in E$ for all $k$.

Because of the periodicity of $p_{E}$, the process $\xi_{n}$ defined by

$$
\xi_{n}=\pi_{T}\left(X_{n}\right)
$$

is a Markov process on $T$ with transition probability $p_{T}$ given by

$$
p_{T}(\xi, \eta)=\sum_{v: \pi_{T}(v)=\eta} p_{E}(\xi, v) .
$$

The same periodic graph may be expressed by another (additive) group, say $\Gamma^{\prime}$ (with an associated $T^{\prime}$ ). In such a case any group containing $\Gamma \cup \Gamma^{\prime}$ serves as one, although a maximal one is usually chosen. The process $\xi_{n}$ as well as $p_{T}$ depends on the choice of $\Gamma$, while all the arguments given below will apply to each of them.

For simplicity, the fundamental set $T$ is supposed to be finite, so that the Markov process $\xi_{n}$ possesses very nice ergodicity with an invariant probability $\mu$ since, from the irreducibility of the walk $X_{n}$, that of $\xi_{n}$ follows. (When $T$ is infinite it suffices to suppose the process $\xi_{n}$ to satisfy Doeblin's condition (cf. [12]).) One sees that

$$
m(u):=\mu\left(\pi_{T}(u)\right)
$$

is an invariant measure of the process $X_{n}$; it is positive and invariant under the translations by $\gamma \in \Gamma$. The process is called symmetric if $m(u) p_{E}(u, v)=$ $m(v) p_{E}(v, u)$ for every pair $u, v \in V$.

The process $\left(\xi_{n}, X_{n}\right)$ may be viewed as a Markov additive process on $T \times \mathbf{R}^{d}$ in the sense that the law of the increment

$$
Y_{1}:=X_{1}-X_{0}
$$

is determined by the value of $\xi_{0}=\pi_{T}\left(X_{0}\right)$; in other words, given $\xi_{0}, Y_{1}$ is conditionally independent of $X_{0}$. Set

$$
P_{\xi}[\cdot]=P\left[\cdot \mid X_{0}=\xi\right] \quad \text { for } \xi \in T ; \text { and } P_{\mu}=\sum_{\xi \in T} \mu(\xi) P_{\xi},
$$

and denote by $E_{\xi}$ and $E_{\mu}$ the corresponding expectations. We suppose that

$$
E_{\mu}\left[Y_{1}\right]=0
$$

which condition corresponds to the zero mean condition in the case of classical random walks made up of sums of i.i.d. random variables ([2]). It will also be supposed that $E_{\mu}\left|Y_{1}\right|^{k+\delta}<\infty$ for some $k \geq 2, \delta \geq 0$. In this paper we derive under 
these assumptions asymptotic expansions of the Green function and the transition probability of $X_{n}$ by applying corresponding or related results for Markov additive processes given in [12. By applying the former, we compute the asymptotic form of the harmonic measure on a half space for a class of walks. To state our results we need more notation.

Let $\mathbf{p}$ stand for the Markovian operator given by the kernel $p_{T}$ : $\mathbf{p} f(\xi)=$ $\sum_{\eta \in T} p_{T}(\xi, \eta) f(\eta)$, and define the vector valued functions $h$ and $c$ on $T$ by

$$
h(\xi)=E_{\xi}\left[Y_{1}\right], c=(1-\mathbf{p})^{-1} h,
$$

where by the latter it is meant that $c$ is a unique solution of $(1-\mathbf{p}) c=h$ such that $\langle\mu, c\rangle:=\sum_{\xi \in T} \mu(\xi) c(\xi)=0$; and we shall also make use of their adjoints

$$
h^{*}(\xi)=E_{\mu}\left[-Y_{1} \mid \xi_{1}=\xi\right], c^{*}=\left(1-\mathbf{p}^{*}\right)^{-1} h^{*},
$$

where the kernel of $\mathbf{p}^{*}$ is given by $p_{T}^{*}(\xi, \eta)=\mu(\eta) p_{T}(\eta, \xi) / \mu(\xi)$. Both $c$ and $c^{*}$ are well defined since $\langle\mu, h\rangle=\left\langle\mu, h^{*}\right\rangle=0$.

It is natural to consider $\tilde{Y}_{n}:=Y_{n}-c\left(\xi_{n-1}\right)+c\left(\xi_{n}\right)$ as an increment in place of $Y_{n}=X_{n}-X_{n-1}$. In fact, the corresponding walk $X_{0}+\tilde{Y}_{1}+\cdots+\tilde{Y}_{n}$ differs from the original $X_{n}$ merely by $c\left(\xi_{n}\right)-c\left(\xi_{0}\right)$, while $\tilde{Y}_{1}$ is better centralized so that for every $\xi \in T$,

$$
E_{\xi}\left[\tilde{Y}_{1}\right]=h(\xi)-c(\xi)+\mathbf{p} c(\xi)=0
$$

From this we may conclude that the central limit theorem variance for the walk $X_{n}$, common for the sums of $\tilde{Y}_{n}$, must equal the symmetric matrix $Q$ whose quadratic form, denoted by $Q(\theta)$, is

$$
Q(\theta)=\theta \cdot Q \theta=E_{\mu}\left(\theta \cdot\left(Y_{1}-c\left(\xi_{0}\right)+c\left(\xi_{1}\right)\right)\right)^{2} .
$$

To be precise, with the help of the Schwarz inequality, we infer that for each $\xi \in T$,

$$
\frac{1}{n} E_{\xi}\left(\theta \cdot X_{n}\right)^{2}=\frac{1}{n} \sum_{k=1}^{n} E_{\xi}\left(\theta \cdot \tilde{Y}_{k}\right)^{2}+O(1 / \sqrt{n}) \longrightarrow Q(\theta)
$$

as $n \rightarrow \infty$, in particular $Q$ does not depend on the choice of $\Gamma$. As is easily checked, if we define two symmetric matrices $Q^{\circ}$ and $R$ by

$$
Q^{\circ}(\theta):=E_{\mu}\left(\theta \cdot Y_{1}\right)^{2} \text { and } R(\theta)=2\left\langle\mu,\left(h^{*} \cdot \theta\right)(c \cdot \theta)\right\rangle,
$$

then $Q(\theta)=Q^{\circ}(\theta)-R(\theta)$. It may well be noticed that $Q=Q^{\circ}$ if either $h=0$ or $h^{*}=0$, and that if $Q=Q^{\circ}$ and $Q$ is isotropic, then

$$
Q(\theta)=\left(d^{-1} E_{\mu}\left|Y_{1}\right|^{2}\right)|\theta|^{2} .
$$

Here $|\theta|:=\sqrt{\theta_{1}^{2}+\cdots+\theta_{d}^{2}}$ (the Euclidian length of $\theta \in \mathbf{R}^{d}$ ). We shall compute $Q, c$ and $c^{*}$ explicitly for various examples (Section 5), of which many satisfy the condition $h=0$ or $h^{*}=0$.

Further define

$$
\sigma=(\operatorname{det} Q)^{1 / 2 d},\|w\|=\sigma \sqrt{w \cdot Q^{-1} w} .
$$

If $Q$ is isotropic, namely $Q(\theta)=\sigma^{2}|\theta|^{2}$, then $\|w\|$ agrees with $|w|$. Let $A$ denote the matrix formed by column vectors $e_{1}, \ldots, e_{d}$ :

$$
A=\left[e_{1} e_{2} \ldots e_{d}\right] \text {. }
$$


Finally set

$$
K_{u, v}=Q^{-1}\left(c\left(\pi_{T}(u)\right)-c^{*}\left(\pi_{T}(v)\right)\right) .
$$

The Green functions. Denote by $p_{E}^{n}$ the $n$-step transition probability of the walk $\left.\overline{X_{n}: p_{E}^{n}(u, v)=P\left[X_{n}\right.}=v \mid X_{0}=u\right]$. In the dimensions $d \geq 3$ the Green function $G(u, v)$ is defined as usual by

$$
G(u, v)=\sum_{n=0}^{\infty} p_{E}^{n}(u, v) \quad(d \geq 3) .
$$

Here we give a result on $G$ under a particular moment condition on $X_{1}$ for simplicity (see [12] for other cases).

Theorem 1.1. Let $d \geq 3$. If

$$
E_{\mu}\left[\left|X_{1}\right|^{4}\right]<\infty(d=3) ; E_{\mu}\left[\left|X_{1}\right|^{4}|\log | X_{1} \mid\right]<\infty(d=4) ; E_{\mu}\left[\left|X_{1}\right|^{d}\right]<\infty(d \geq 5),
$$

then, on writing $w=v-u$,

$$
\frac{G(u, v)}{m(v)|\operatorname{det} A|}=\frac{\kappa_{d}}{\sigma^{2}\|w\|^{d-2}}+\left[\frac{U(w)}{\|w\|^{d+2}}+\frac{(d-2) \kappa_{d} w \cdot K_{u, v}}{\|w\|^{d}}\right]+O\left(\frac{1}{|w|^{d}}\right)
$$

as $|w| \rightarrow \infty$, where $\kappa_{d}=\pi^{-d / 2}(d-2)^{-1} \Gamma\left(\frac{1}{2} d\right)$; and $U(w)$ is a homogeneous polynomial of degree three (with coefficients independent of $\pi_{T}(u)$ and $\pi_{T}(v)$ ) and vanishes if the process is symmetric.

For $d=2$, we define the function $G(u, v)$ by

$$
\frac{G(u, v)}{m(v)}=\sum_{n=0}^{\infty}\left(\frac{p_{E}^{n}(u, v)}{m(v)}-\frac{p_{E}^{n}(u, u)}{m(u)}\right) \quad(d=2) .
$$

Let $\gamma$ be Euler's constant and set for $\xi \in T$,

$$
\lambda(\xi)=\frac{\log \sqrt{2 \sigma^{2}}-\gamma}{\pi \sigma^{2}}-\sum_{n=0}^{\infty}\left(\frac{p_{E}^{n}(\xi \cdot \xi)}{\mu(\xi)|\operatorname{det} A|}-\frac{\mathbf{1}(n>0)}{2 \pi \sigma^{2} n}\right) .
$$

Theorem 1.2. Let $d=2$. Suppose that $E_{\mu}\left|Y_{1}\right|^{4}<\infty$. Then, on writing $w=v-u$,

$$
\frac{G(u, v)}{m(v)|\operatorname{det} A|}=-\frac{1}{\pi \sigma^{2}} \log \|w\|+\lambda\left(\pi_{T}(u)\right)+\left[\frac{U(w)}{\|w\|^{4}}+\frac{w \cdot K_{u, v}}{\pi\|w\|^{2}}\right]+r(u, v)
$$

as $|w| \rightarrow \infty$. Here $r(u, v)$ is the remainder term which is of the order $O\left(|w|^{-2}\right)$ and $U$ is similar to the one in Theorem 1.1. (See (15) for more details on $r(u, v)$.)

Remark (i). It is reasonable to have $m(v)|\operatorname{det} A|$ in the denominator on the left hand side of (5). This is because, on the one hand, the mass $m(v)$ represents the relative frequency of how often $X_{n}$ visits a vertex $v$ among those of $T+\pi^{\Gamma}(v)$ $\left(\pi^{\Gamma}(v):=v-\pi_{T}(v)\right)$ and, on the other hand, $|\operatorname{det} A|^{-1}$ equals the density of points of $\Gamma$ in $\mathbf{R}^{d}$ relative to the Euclidian metric of $\mathbf{R}^{d}$; hence $m(v)|\operatorname{det} A|$ may be regarded as a natural weight of the vertex $v$.

Remark (ii). The formula (5) reveals that $m(v)|\operatorname{det} A|, U(v-u), c\left(\pi_{T}(u)\right)$ and $c\left(\pi_{T}(v)\right)$ do not depend on the choice of $\Gamma$ (for verification recall that $\langle\mu, c\rangle=$ $\left.\left\langle\mu, c^{*}\right\rangle=0\right)$, a fact that may be shown in more or less direct arguments. 
While in Theorems 1.1 and 1.2 we have not made any additional assumptions on periodicity of the process, the local central limit theorem is sensitive to it.

Local central limit theorems. Denote by $s$ the period of the walk $X_{n}$, namely $s$ stands for the period of the semi-group $\left\{n>0: p_{E}^{n}(u, u)>0\right\}$ for any $u \in V$ (s is independent of $u$ ). If $s=1$, then, for each $u \in V$, there exists a number $n_{\circ}=n_{\circ}(u)$ such that $p_{E}^{n}(u, u)>0$ for all $n \geq n_{\circ}$. If $s>1, V$ is partitioned into subsets $V_{0}, \ldots, V_{s-1}$ so that if $u \in V_{j}$ and $v \in V_{k}$, then $p_{E}^{m s+k-j}(u, v)>0$ for all sufficiently large $m$, and $p_{E}^{n}(u, v)=0$ for all $n \neq k-j(\bmod s)$. We call the process $X_{n}$ aperiodic if $s=1$ and periodic otherwise. In what follows we set $V_{j+m s}=V_{j} ; \mathbf{1}(S)$ will stand for the indicator of a statement $S$.

Theorem 1.3. Suppose that $E_{\mu}\left[\left|Y_{1}\right|^{k+\delta}\right]<\infty$ with some $k \geq 2$ and $\delta \in[0,1)$. If $u \in V_{j}$ and $n=m s+\ell(\ell=0, \ldots, s-1, m=1,2, \ldots)$, then

$$
\begin{aligned}
\frac{\left(2 \pi \sigma^{2} n\right)^{d / 2} p_{E}^{n}(u, v)}{m(v)|\operatorname{det} A|}= & \exp \left(-\frac{\|w\|^{2}}{2 \sigma^{2} n}\right)\left[1+P^{n, k}(w)\right] s \mathbf{1}\left(v \in V_{j+\ell}\right) \\
& +o\left(\frac{1}{\sqrt{n}^{k-2+\delta}} \wedge \frac{n}{|w|^{k+\delta}}\right),
\end{aligned}
$$

as $n \rightarrow \infty$ uniformly in $v \in V$, where $w=v-u$; $a \wedge b$ stands for the minimum of $a$ and $b ; P^{n, k}(y)$ is a polynomial of $y \in \mathbf{R}^{d}$ such that $P^{n, 2} \equiv 0$ and if $k \geq 3$

$$
P^{n, k}(y)=\frac{1}{\sqrt{n}} P_{1}^{\xi, \eta}\left(\frac{y}{\sqrt{n}}\right)+\cdots+\frac{1}{\sqrt{n}^{k-2}} P_{k-2}^{\xi, \eta}\left(\frac{y}{\sqrt{n}}\right),
$$

where $\xi=\pi_{T}(u), \eta=\pi_{T}(v)$, and $P_{i}^{\xi, \eta}(y)$ is a polynomial (with coefficients depending on $\xi$ as well as $\eta$ but independent of $n$ ) of degree at most $3 i$ and an odd or even function depending on whether $i$ is odd or even. The first polynomial $P_{1}^{\xi, \eta}$ is of the form

$$
P_{1}^{\xi, \eta}(y)=H(y)+y \cdot Q^{-1}\left[c(\xi)-c^{*}(\eta)\right],
$$

where $H$ is an odd polynomial of degree at most three and identically zero if the process is symmetric.

The local central limit theorem for symmetric walks on periodic graphs is studied from a geometrical point of view by Kotani, Shirai and Sunada [6] (cf. also [7], 8]), where the covariance matrix $Q$ is described in a quite different way. The principal order term in the expansion (6) can be readily derived from the corresponding results for Markov additive processes that are previously obtained (cf. [3], [5], 4]) with a different expression of $Q$ from ours (see Section 3 ). In the case when $k=2$ and $\delta=0$ and the walk is aperiodic in the sense that the condition (7) below holds with $\tau=1$, Theorem 1.3 is proved in a somewhat different approach by Takenami 9] (also with a different expression of $Q$ ).

Remark (iii). We call the process $\xi_{n}$ cyclic (instead of 'periodic', a more usual word) if $T$ is partitioned into cyclically moving sets $T_{0}, \ldots, T_{\tau-1}(\tau \geq 2)$ so that $p_{T}\left(\xi, T_{j}\right)=\mathbf{1}\left(\xi \in T_{j-1}\right), j=1, \ldots, \tau$, where $T_{\tau}:=T_{0}$. If the process $\xi_{n}$ is not cyclic, let $\tau=1$ and $T_{0}=T$. If $\tau>1$ and

$$
p_{E}^{n \tau}(u, u)>0 \text { for } u \in V \text { for all sufficiently large } n,
$$

then the process $X_{n}$ is periodic with the period $s=\tau$ and $V_{k}=\left\{u \in V: \pi_{T}(u) \in\right.$ $\left.T_{k}\right\}$. This is a rather special case of periodic walks. In general, $s$ may be greater 
than $\tau$ (see Examples 4 and 6 of Section 5); in fact $s=\tau$ if and only if (7) is true since the set $\left\{n: p_{E}^{n \tau}(u, u)>0\right\}$ is a semi-group for each $u \in V$.

Walks on a half space. The second order term of the Green function is significant for finding out the principal term of the Green function or harmonic measure of the process on a half space with absorbing boundary. To illustrate this point we consider the case when the process is symmetric relative to a hyperplane and continuous downward. Let $M \subset \mathbf{R}^{d}$ be a $d-1$ dimensional plane passing through the origin and let $e$ be a unit normal vector of $M$. We decompose $V \backslash M$ into positive and negative halves, $V^{+}$and $V^{-}$say: $V^{ \pm}=\{u \in V: \pm u \cdot e>0\}$ and set $V_{0}=V \cap M$. Suppose that

(a) the law of the walk $X_{n}$ is mirror symmetric relative to $M$,

(b) $V_{0}$ separates $V^{-}$from $V^{+}$(downwardly).

By (b) we mean that every path of $(V, E)$ started at a point of $V^{+}$and ending at a point of $V^{-}$must contain at least one vertex of $V_{0}$. Let $G^{+}(u, v)$ denote the Green function of the walk $X_{n}$ starting at a point of $V^{+}$and killed as it hits $V_{0}$. (For the precise meaning of (a) see $\mathbf{4 . 6}$ of Section 4 if necessary.) Under (a) and (b) the reflection principle works, so that if $\bar{v}$ denotes the mirror symmetric point of $v$, then for $u, v \in V^{+}$,

$$
G^{+}(u, v)=G(u, v)-G(u, \bar{v}) .
$$

Theorem 1.4. Suppose that $E_{\mu}\left[\left|X_{1}\right|^{5}\right]<\infty(d=2,3) ; E_{\mu}\left[\left|X_{1}\right|^{5}\left|\log X_{1}\right|\right]<\infty$ $(d=4) ; E_{\mu}\left[\left|X_{1}\right|^{d+1}\right]<\infty(d \geq 5)$ and that the conditions (a) and (b) above are satisfied. Let $u, v \in V^{+}$and put $\xi=\pi_{T}(u)$ and $\eta=\pi_{T}(v)$. Then

$$
\begin{aligned}
\frac{G^{+}(u, v)}{m(v)|\operatorname{det} A|}= & \frac{2 \Gamma(d / 2)}{\pi^{d / 2} Q(e)} \cdot \frac{[(u+c(\xi)) \cdot e]\left[\left(v+c^{*}(\eta)\right) \cdot e\right]}{\|v-u\|^{d}} \\
& \times\left\{1+O\left(\frac{1}{|v-u|}+\frac{(u \cdot e)(v \cdot e)}{|v-u|^{2}}\right)\right\},
\end{aligned}
$$

as $|v-u| \rightarrow \infty$ in such a manner that $(u \cdot e)(v \cdot e) /|u-v|^{2} \rightarrow 0$.

Let $H(u, s)\left(u \in V^{+}, s \in V_{0}\right)$ denote the harmonic measure (hitting distribution of $V_{0}$ ) for the process started at $u$ and stopped on $V_{0}$. Then under (a) and (b)

$$
H(u, s)=\sum_{v \in V^{+}} G^{+}(u, v) p_{E}(v, s)
$$

From Theorem 1.4 we can easily deduce the following estimate of $H$.

Corollary 1.1. Suppose that the same conditions on $X_{n}$ as imposed in Theorem 1.4 are satisfied. Let $u \in V^{+}, s \in V_{0}$ and put $\xi=\pi_{T}(u)$ and $\eta=\pi_{T}(s)$. Then, as $|u-s| \rightarrow \infty$,

$$
H(u, s)=\frac{\Gamma(d / 2)}{\pi^{d / 2}} \cdot \frac{(u+c(\xi)) \cdot e}{\|u-s\|^{d}} L(\eta)+O\left(\frac{|u \cdot e|+1}{|u-s|^{d+1}}\right) \quad(d \geq 2),
$$

where, with the dual $p_{E}^{*}$ of $p_{E}$ defined by $\mu(\eta) p_{E}^{*}(s, v)=\mu\left(\pi_{T}(v)\right) p_{E}(v, s)$,

$$
L(\eta)=\frac{2|\operatorname{det} A| \mu(\eta)}{Q(e)} \sum_{v \in V^{+}}\left[\left(v+c^{*}\left(\pi_{T}(v)\right)\right) \cdot e\right] p_{E}^{*}(s, v) .
$$


(Owing to the periodicity of $p_{E}$ the last sum depends on $s \in V_{0}$ only through $\eta ; p_{E}^{*}$ is the transition probability of the dual walk (see Section 4.1).)

By (a) and (b) we can choose the frame $A=\left[e_{1}, \ldots, e_{d}\right]$ so that $e_{1}, \ldots, e_{d-1} \in M$. With this choice of $A$ we define

$\mathrm{v}=$ 'the volume of the $(d-1)$-dimensional parallelogram spanned by $e_{1}, \ldots, e_{d-1}$ '. Set $T_{0}=\left\{\eta \in T: \eta=\pi_{T}(s)\right.$ for some $\left.s \in V_{0}\right\}$. Since $H(u, \cdot)$ is a probability measure for every $u \in V^{+}$, from the formula in Corollary 1.1 we obtain the curious identity

$$
\sum_{\eta \in T_{0}} L(\eta)=\mathrm{v}
$$

which we do not know how to verify by direct computation. If $d=2$ and $\sharp T_{0}=1$ (namely $T_{0}$ consists of a single vertex), then $\mathrm{v}$ equals the span of the one-dimensional lattice $V_{0}$. From the identity (9) one can immediately find out the value of $L(\eta)$ in the mirror symmetric cases of Examples 3.1, 4, 5.3, 6.1, 6.2 of Section 5.

Remark (iv). Theorem 1.4 reveals that the Martin (exit) boundary of the process killed on $V_{0}$ consists of a single point, i.e., the regular function $\left(u+c\left(\pi_{T}(u)\right)\right) \cdot e$ (uniqueness is up to multiplicative constants). Without the symmetry property (a) but with the assumption (b), a simple computation shows that the function

$$
f(u)=\left(u+c\left(\pi_{T}(u)\right)\right) \cdot e-\sum_{s \in V_{0}} H(u, s) c\left(\pi_{T}(s)\right) \cdot e \quad\left(u \in V^{+}\right)
$$

is a regular function of the transition kernel $p_{E}$ restricted to $V^{+}$. The uniqueness might be proved when $\sharp T_{0}=1$ since in that case we can compute an asymptotic form of $H$ and thereby that of $G^{+}$(the computation is rather involved).

For another application of Theorem 1.4 let $q^{+}(u, v)\left(u, v \in V^{+}\right)$denote the probability that the walk started at $u$ visits $v$ at some positive time before it hits $V_{0}$, and set $E^{+}(u)=1-q^{+}(u, u)$ (escape probability). It then follows that

$$
q^{+}(u, v)=G^{+}(u, v) E^{+}(v)=G^{+}(u, v) / G^{+}(v, v) .
$$

Thus Theorem 1.4 yields asymptotic estimates of $q^{+}$as $|v-u| \rightarrow \infty$ in a suitable way. (By (5) $G^{+}(v, v) \sim\left(\pi \sigma^{2}\right)^{-1}|\operatorname{det} A| m(v) \log (v \cdot e)$ as $v \cdot e \rightarrow \infty$ if $d=2$.)

We conclude this section by pointing out another way of viewing $X_{n}$. Fix $\xi^{\circ} \in T$ arbitrarily and let $\tau_{n}$ be the successive times when $\xi_{n}$ visits $\xi^{\circ}$. Then the imbedded process $\tilde{X}_{n}:=X_{\tau_{n}}$ is an ordinary random walk on $\Gamma$ with independent increments; if $\tilde{Q}$ is the covariance matrix of $\tilde{X}_{1}-\tilde{X}_{0}$, then $Q=\mu\left(\xi^{\circ}\right) \tilde{Q}$ (which is often useful for computing $Q$ ); and for $u, v$ such that $\pi_{T}(u)=\pi_{T}(v), G(u, v)$ agrees with the Green function of $\left(\tilde{X}_{n}\right)$, whose asymptotics is known (cf. [1]), although this does not provide second-order terms as in (4) or (5).

The rest of the paper is organized as follows. In Section 2 we introduce a Markov additive process which is somewhat different from $\left(\xi_{n}, X_{n}\right)$, and prove Theorems 1.1. 1.2 and 1.3. In Section 3 the quantities for the Markov additive process introduced in Section 2 are related to those defined in Section 1. In Section 4 we discuss some symmetries of the walks. In Section $5 Q$ and $c$ are explicitly computed for many examples. In Section 6 we prove Theorem 1.4 . 


\section{Proof of Theorems 1.1, 1.2 and 1.3}

To the random walk $X_{n}$ there is naturally associated a Markov additive process on $T \times \mathbf{Z}^{d}$, to which we can directly apply the previous results (cf. [1], 3], [5, 4], [12], etc.) as will be done for the asymptotic expansion of $G$ in the next section. In this section we prove Theorems 1.1 and 1.2 with the help of this process but not by a direct application of such results; instead we go back to a Fourier representation formula for the Green function or the transition probability of $X_{n}$. In this approach the expansions are (directly) given in the original coordinate system with simpler expressions of both $Q$ and the second order term (compare it with those given in the next section).

A stochastic process $\left(\eta_{n}, S_{n}\right)(n=0,1,2, \ldots)$ taking values in the product space $T \times \mathbf{Z}^{d}$ is a Markov additive process if it is a time homogeneous Markov process on the state space $T \times \mathbf{Z}^{d}$ whose one step transition law is such that the conditional distribution of $\left(\eta_{n}, S_{n}-S_{n-1}\right)$ given $\left(\eta_{n-1}, S_{n-1}\right)$ does not depend on the value of $S_{n-1}$.

A Markov additive process on $T \times \mathbf{Z}^{d}$ is induced from $X_{n}$ as follows. Recalling that $\gamma_{x}=A x$, where $A=\left[e_{1} e_{2} \ldots e_{d}\right]$ as defined previously, we find the random variables $\hat{S}_{n} \in \mathbf{Z}^{d}$ and $\xi_{n} \in T$ to be uniquely determined by the relation

$$
X_{n}=A \hat{S}_{n}+\xi_{n} .
$$

( $\xi_{n}$ is the same as the one introduced in Section 1.) It is readily seen that $\left(\xi_{n}, \hat{S}_{n}\right)$ is a Markov additive process on $T \times \mathbf{Z}^{d}$.

The following condition is introduced in [12].

Condition (AP). We say a MA process $\left(\xi_{n}, S_{n}\right)$ on $T \times \mathbf{Z}^{d}$ satisfies Condition $(A P)$ (or simply $(A P)$ ) if there exists no proper subgroup $H$ of the additive group $\mathbf{Z}^{d}$ such that

$$
\forall n \geq 1, P_{\mu}\left[\exists a \in \mathbf{Z}^{d}, P_{\mu}\left[S_{n} \in H+a \mid \sigma\left\{\xi_{0}, \xi_{n}\right\}\right]=1\right]=1 .
$$

$\left(\sigma\left\{\xi_{0}, \xi_{n}\right\}\right.$ denotes the $\sigma$-fields generated by $\xi_{0}$ and $\xi_{n}$.)

If the walk $X_{n}$ is aperiodic or (17) holds in the case $\tau>1$, then the MA process $\left(\xi_{n}, \hat{S}_{n}\right)$ induced from $X_{n}$ as above satisfies (AP) and vice versa. (See Proposition 2.1 below.)

Proof of Theorem 1.1. We adapt the proof of Theorem 9 in [12. First we suppose that the MA process $\left(\xi_{n}, \hat{S}_{n}\right)$ defined above satisfies Condition (AP). If $u=A x_{0}+\xi$ and $v=A x+\eta$ with $\xi, \eta \in T, x_{0}, x \in \mathbf{Z}^{d}$,

$$
\begin{aligned}
p_{E}^{n}(u, v) & =P\left[\hat{S}_{n}=x, \xi_{n}=\eta \mid X_{0}=u\right] \\
& =\frac{1}{(2 \pi)^{d}} \int_{[-\pi, \pi)^{d}} E\left[e^{i \hat{S}_{n} \cdot \theta} ; \xi_{n}=\eta \mid \hat{S}_{0}=x_{0}, \xi_{0}=\xi\right] e^{-i \theta \cdot x} d \theta .
\end{aligned}
$$

Changing the variable according to $\theta={ }^{t} A \theta^{\prime}$, setting $\Delta^{\prime}=\left\{\theta^{\prime}:{ }^{t} A \theta^{\prime} \in[-\pi, \pi)^{d}\right\}$ and recalling $\hat{S}_{n}=A^{-1}\left(X_{n}-\xi_{n}\right)$, we can rewrite the right side as

$$
\frac{|\operatorname{det} A|}{(2 \pi)^{d}} \int_{\Delta^{\prime}} E\left[e^{i\left(X_{n}-X_{0}\right) \cdot \theta^{\prime}} ; \xi_{n}=\eta \mid X_{0}=u\right] e^{-i \theta^{\prime} \cdot(v-u)} d \theta^{\prime} .
$$

To carry out the Fourier integration we employ the last expression on some $\varepsilon$ neighborhood of $\theta=0$ and (12) on the rest. The contribution of the latter is negligible owing to Condition (AP) (cf. Section 7 of [12]). As for that of the 
former we make some perturbation arguments (as given in [3], 5]) to evaluate $E\left[e^{i\left(X_{n}-X_{0}\right) \cdot \theta^{\prime}} ; \xi_{n}=\eta \mid X_{0}=u\right]$ about the origin of $\theta^{\prime}$-space so that usual methods for Fourier integration are applicable (cf. [12] (Section 2), [10, 11], 1], etc.). These will lead to the desired estimates of $G(u, v)$.

If $\left(\xi_{n}, \hat{S}_{n}\right)$ does not satisfy Condition (AP), we can choose a minimal subgroup $H$ for which (11) holds. Consider the quotient group $K=\mathbf{Z}^{d} / H$ and define the processes $a_{n}$ on $K, \breve{S}_{n}$ on $H$ and $\breve{\xi}_{n}$ on $T \times K$ by

$$
\hat{S}_{n}=\breve{S}_{n}+a_{n} \text { and } \breve{\xi}_{n}=\left(\xi_{n}, a_{n}\right) .
$$

Then $\left(\breve{\xi}_{n}, \breve{S}_{n}\right)$ is a MA process on $(T \times K) \times H$, of which Condition (AP) is valid (Proposition 8 of [12]) and the first component $\breve{\xi}_{n}$ is ergodic (owing to the irreducibility of $X_{n}$ ), and we can proceed as above. The details are omitted.

A similar argument to the above shows Theorem 1.2 (cf. Corollary 3 of [12]).

Proof of Theorem 1.3. We can proceed in a similar way as above but applying (if necessary) the following proposition (see also a remark given just after its proof).

Proposition 2.1. For Condition (AP) to be satisfied by $\left(\xi_{n}, \hat{S}_{n}\right)$ it is necessary and sufficient that the condition (7) holds, or, what amounts to the same, there exists a positive integer $n^{\circ}$ (necessarily a multiple of $\tau$ ) such that for each $k=0, \ldots, \tau-1$,

$$
p_{E}^{n^{\circ}}(\xi, \eta)>0 \text { for all } \xi, \eta \in T_{k} .
$$

Proof. If $\left(\xi_{n}, \hat{S}_{n}\right)$ satisfies Condition (AP), then $s=\tau$ according to a local central limit theorem valid under (AP) (cf. Theorem 4 of [12]). This proves the necessity part (see Remark (iii) in Section 1). For sufficiency suppose that (14) holds. Then by an elementary argument for Markov chains we see that $p^{m \tau+\ell}(\xi, \eta)>0$ for all $\xi \in T_{0}, \eta \in T_{\ell}$ and for all sufficiently large $m$. Since $\hat{S}_{n}=0$ if $X_{n} \in T$, we infer that for all sufficiently large $n, P_{\xi}\left[\hat{S}_{n}=0 \mid \sigma\left\{\xi_{n}\right\}\right]>0\left(P_{\xi}\right.$-a.s. $)$, hence $P_{\mu}\left[\hat{S}_{n} \in H\right]=1$ if (11) holds for a subgroup $H$ and the walk $\hat{S}_{n}$. The last relation is impossible unless $H=\mathbf{Z}^{d}$ owing to the irreducibility of $X_{n}$. Thus Condition (AP) is satisfied by $\left(\xi_{n}, \hat{S}_{n}\right)$.

Validity of (14) as well as (AP) may depend on the choice of $\Gamma$. From Proposition 2.1 we can easily deduce that there exists a frame $\Gamma$ so that $\left(\xi_{n}, \hat{S}_{n}\right)$ satisfies Condition (AP).

\section{The expansion of the Green function for $\left(\xi_{n}, \hat{S}_{n}\right)$}

We relate the expansion of the Green function of $X_{n}$ to that of the Markov additive process $\left(\xi_{n}, \hat{S}_{n}\right)$ on $T \times \mathbf{Z}^{d}$ (defined in (10)), of which it is well understood that the Fourier method applies as in the case of usual random walks ([1], [3], [5], 4], 12]), and thereby provides another proof of (a weak version of) Theorem 1.1. The expressions of $Q$ and $c$ obtained in this context would be useful in some circumstances.

Define functions $\hat{h}$ and $\hat{c}$ on $T$ by

$$
\hat{h}(\xi)=E\left[\hat{S}_{1} \mid X_{0}=\xi\right], \hat{c}(\xi)=\left(1-p_{T}\right)^{-1} \hat{h}(\xi) \quad(\xi \in T),
$$


and a symmetric matrix $\hat{Q}$ by means of quadratic form:

$$
\hat{Q}(\theta):=\theta \cdot \hat{Q} \theta=E_{\mu}\left(\theta \cdot\left(\hat{S}_{1}-\hat{c}\left(\xi_{0}\right)+\hat{c}\left(\xi_{1}\right)\right)\right)^{2} \quad\left(\theta \in \mathbf{R}^{d}\right),
$$

and set $\hat{\sigma}=(\operatorname{det} \hat{Q})^{1 / 2 d}$. Further define the adjoints $\hat{h}^{*}$ and $\hat{c}^{*}$ analogously to $h^{*}$ and $c^{*}$.

Let $d=2$. Suppose that $E_{\mu}\left[\left|\hat{S}_{1}\right|^{2+m+\delta}\right]<\infty$ for some $\delta \in(0,1)$ and an integer $m \geq 1$. Then the function $G$ admits the following asymptotic expansion: for $u=\xi+A y, v=\eta+A y+A x(\xi, \eta \in T)$,

$$
\begin{aligned}
\frac{G(u, v)}{\mu(\eta)}= & -\frac{\log \|x\|_{\wedge}}{\pi \hat{\sigma}^{2}}+\hat{\lambda}(\xi)+\left[\frac{\hat{U}(x)}{\|x\|_{\wedge}^{4}}+\frac{\left(\hat{Q}^{-1} x\right) \cdot\left(\hat{c}(\xi)-\hat{c}^{*}(\eta)\right)}{\pi\|x\|_{\wedge}^{2}}\right] \\
& +\left[\frac{\left\{x^{6}\right\}}{\|x\|_{\wedge}^{8}}+\cdots+\frac{\left\{x^{3 m}\right\}}{\|x\|_{\wedge}^{4 m}}+\frac{R_{m}(x, \xi, \eta)}{|x|^{m+\delta}}\right] \quad(x \neq 0),
\end{aligned}
$$

with $\lim _{|x| \rightarrow \infty} \sup _{\xi} \int\left|R_{m}(x, \xi, \eta)\right| \mu(d \eta)=0$. Here $\|x\|_{\wedge}=\hat{\sigma} \sqrt{x \cdot \hat{Q}^{-1} x}, \hat{U}(x)$ is a homogeneous polynomial of degree 3 (with coefficients independent of variables $\xi, \eta$ ) and vanishes if the process is symmetric and $\left\{x^{j}\right\}$ stands for some homogeneous polynomial in $x \in \mathbf{R}^{2}$ of degree $j$. (See Theorem 2 of [12].)

The expression on the right side of (15) depends on the choice of the fundamental set $T$ as well as the frame $\Gamma$. In order to write it down independently of the choice of $T$ or $\Gamma$ we introduce some notation. First define

$$
Q_{V}=A \hat{Q} A^{t} .
$$

Then for $u=\xi+\gamma_{y}, v=\eta+\gamma_{y+x}$ as above, writing $w=v-u$, we have

$$
\|x\|_{\wedge}^{2}=\hat{\sigma}^{2} \gamma_{x} \cdot Q_{V}^{-1} \gamma_{x}=\hat{\sigma}^{2}(w-(\eta-\xi)) \cdot Q_{V}^{-1}[w-(\eta-\xi)],
$$

so that as $|w| \rightarrow \infty$,

$$
\|x\|_{\wedge}=\frac{1}{|\operatorname{det} A|^{1 / d}}\|w\|_{V}\left[1-\frac{\sigma_{V}^{2} Q_{V}^{-1} w \cdot(\eta-\xi)}{\|w\|_{V}^{2}}+O\left(\frac{1}{|w|^{2}}\right)\right],
$$

where $\sigma_{V}=\left(\operatorname{det} Q_{V}\right)^{1 / 2 d}=\hat{\sigma}|\operatorname{det} A|^{1 / d}$ and $\|w\|_{V}=\sigma_{V} \sqrt{w \cdot Q_{V}^{-1} w}$. Further put

$$
c_{V}(\xi)=A \hat{c}(\xi), c_{V}^{*}(\xi)=A \hat{c}^{*}(\xi) \text { and } K_{u, v}^{V}=Q^{-1}\left(c_{V}(\xi)-c_{V}^{*}(\eta)-(\xi-\eta)\right) .
$$

Then we translate the formula (15) in terms of $Q_{V}$ and $c_{V}$. Owing to the next lemma, it is expressed in terms of $Q, K_{u, v}$, etc. and we find a formula that agrees with the one given in Theorem 1.1 at least up to $O\left(1 /|w|^{2}\right)$. Set $g=\sum_{\xi \in T} \mu(\xi) \xi$.

Lemma 3.1. Let $Q, c, c^{*}$ and $K_{u, v}$ be as in the introduction. Then

$$
Q(\theta)=Q_{V}(\theta), K_{u, v}=K_{u, v}^{V}, U=U_{V}
$$

in particular $c(\xi)=c_{V}(\xi)-\xi+g, c^{*}(\xi)=c_{V}^{*}(\xi)-\xi+g(\xi, \eta \in T)$ and $\|\cdot\|=$ $\|\cdot\|_{V}=|\operatorname{det} A|^{1 / d}\|\cdot\|_{\wedge}$.

Proof. Although the proof can be done by a direct computation as in 12] (Lemma 12 ), here we proceed in a different way. Since $A \hat{S}_{n}=X_{n}-\xi_{n}$ and $X_{0}=\xi$ a.s. $\left(P_{\xi}\right)$, we have

$$
\sum_{n=0}^{\infty} E_{\xi}\left[e^{i A \hat{S}_{n} \cdot \theta} ; \xi_{n}=\eta\right]=\sum_{n=0}^{\infty} E_{\xi}\left[e^{i\left(X_{n}-X_{0}\right) \cdot \theta} ; \xi_{n}=\eta\right] e^{i(\xi-\eta) \cdot \theta}
$$


We compute each of the two Fourier integrals appearing in this identity to find the asymptotic behavior of them as $|\theta| \rightarrow 0$ up to the order of $o\left(1 /|\theta|^{3}\right)$ that are expressed by means of $Q_{V}, c_{V}, c_{V}^{*}, U_{V}$ for the left side and by $Q, c, c^{*}, U$ for the right side (see Section 2 of [12]). The comparison of the principal order terms gives $Q_{V}=Q$, which together with the comparison of terms of order $O\left(1 /|\theta|^{3}\right)$ in turn shows the other identities.

Let $\hat{X}_{n}=A \hat{S}_{n}$. For later usage we define

$$
Q_{V}^{\circ}(\theta)=E_{\mu}\left|\hat{X}_{1} \cdot \theta\right|^{2}, R_{V}(\theta)=2 \sum_{\xi \in T} \mu(\xi)\left(h_{V}^{*}(\xi) \cdot \theta\right)\left(c_{V}(\xi) \cdot \theta\right),
$$

so that $Q(\theta)=Q_{V}^{\circ}(\theta)-R_{V}(\theta)$. (Recall $\hat{X}_{0}=0$ a.s. $\left(P_{\mu}\right)$.)

\section{Dual WalK AND some Symmetries}

In this section we collect miscellaneous facts that are useful in computing $Q, c$ and $c *$. The arguments are independent of those of Sections 2 and 3.

4.1. Dual walk. The dual walk $\left(X_{n}^{*}, P_{\xi}^{*}\right)$ is a random walk on $(V, E)$ whose transition probability $p_{E}^{*}$ is given by

$$
p_{E}^{*}(v, u)=m(u) p_{E}(u, v) / m(v) .
$$

If $\xi_{n}^{*}=\pi_{T}\left(X_{n}^{*}\right)$, then $p_{T}^{*}(\eta, \xi):=P_{\eta}^{*}\left[\xi_{1}^{*}=\xi\right]=\mu(\xi) p_{T}(\xi, \eta) / \mu(\eta)$, conforming to the notation previously introduced. It is immediate to see that $P_{\xi}^{*}\left[X_{n}^{*}=u\right]=$ $P_{\mu}\left[X_{n}=-u \mid \xi_{n}=\xi\right], h^{*}(\xi)=\int X_{1}^{*} d P_{\xi}^{*}-\xi$, and $Q^{*}$, the covariance matrix for $\left(X_{n}^{*}\right)$, agrees with $Q$.

4.2. Symmetry relative to the invariant measure. The walk $X_{n}$ is called symmetric if it agrees with the dual walk. It is straightforward that the three conditions

(i) $\left(\xi_{n}, \hat{S}_{n}\right)$ is symmetric; (ii) $\left(\xi_{n}, X_{n}\right)$ is symmetric; (iii) $X_{n}$ is symmetric

are equivalent to one another. Here by (i) (or (ii)) it is meant that the twocomponent process $\left(\xi_{n}, \hat{S}_{n}\right)$ (or $\left.\left(\xi_{n}, X_{n}\right)\right)$ is symmetric relative to $\mu \times$ the counting measure on $\mathbf{Z}^{d}$ (respectively $V$ ). Obviously $c^{*}=c$ for symmetric walks.

4.3. Rotation invariance about a point. Let $d=2$ (for simplicity). If the walk is invariant under the rotation through an angle $\neq n \pi(n \in \mathbf{Z}), Q$ is isotropic. This is simply because $Q$ is then invariant under the rotation through the angle.

4.4. Symmetry relative to each vertex. Suppose that the graph is invariant under the rotation by $\pi$ of its vertices, namely for each vertex $v_{0}$ the mapping $v \in R^{d} \mapsto$ $2 v_{0}-v$ transforms the graph onto itself. If, in addition, the probabilities $p_{E}(v, u)$ do not change under this transformation, namely $p_{E}\left(2 v_{0}-v, 2 v_{0}-u\right)=p_{E}(v, u)$, we call the random walk symmetric relative to each vertex. This symmetry implies that $E_{\xi}\left[X_{1}\right]=0$; hence $h=c=0$.

4.4.1. Suppose a random walk on $V$ to be symmetric relative to each vertex. Then its dual process is also symmetric relative to each vertex. In particular $h^{*}=c^{*}=0$; moreover $U(x)=H(x)=0$ for all $x$, so that the second order term in the asymptotic expansion of $G$ vanishes. 
In the case when $d=2$, if the invariance is under the rotation through $2 \pi / 3$ (instead of $\pi$ ), not only do these relations hold true, but also $Q$ is isotropic (so that by (33) $\left.Q(\theta)=\frac{1}{2} E_{\mu}\left|Y_{1}\right|^{2}|\theta|^{2}\right)$.

Proof. Under the assumption of the first assertion $m$ is also symmetric, namely $m(u)=m(2 v-u)$ for each pair $u, v$, and it is immediate to see the required symmetric property of $p_{E}^{*}$. By the symmetry relative to a vertex $v$ we have $U(u-v)=U(v-u)$ and a similar identity for $H$ (which follows from the explicit representation of $U$ and from the asymptotic formula given in Theorem 1.2 , respectively); hence $U \equiv H \equiv 0$ since $U(x)$ and $H$ are odd functions. The proof of the second assertion is similar (use $\mathbf{4 . 3}$ ).

4.4.2. If a walk is symmetric relative to each vertex, it follows that for any pair $\xi, \eta$ from $T$, the walk as well as the graph is invariant under the shift by $2(\eta-\xi)$. In fact for any two vertices $u$ and $v$,

$$
\begin{aligned}
p_{E}(u, v) & =p_{E}(-u+2 \xi,-v+2 \xi) \\
& =p_{E}(u+2(\eta-\xi), v+2(\eta-\xi)),
\end{aligned}
$$

where the symmetries relative to $\xi$ and $\eta$ are applied for the first and second equalities, respectively.

Conversely if for some (and every in consequence) vertex $\xi_{0} \in T$, the walk (as well as the graph) is invariant under the shift by $2\left(\eta-\xi_{0}\right)$ for every $\eta \in T$, then the symmetry relative to each vertex follows from the symmetry relative to $\xi_{0}$, as is easily verified.

It is noted that under the symmetry relative to each vertex the walk $X_{n}$ is symmetric relative to the invariant measure $m$ if and only if its projection $\xi_{n}$ is symmetric relative to $\mu$.

4.5. Given a set of numbers $0<\lambda(\xi) \leq 1(\xi \in T)$ and a random walk on a periodic graph $V$ with periodic structure $\left(T, A, p_{E}\right)$ such that $p_{E}(u, u)=0$ for all $u \in V$, we consider a new random walk, denoted by $X_{n}^{\lambda}$, on $V$ with the transition law $p_{E}^{\lambda}$ defined by

$$
p_{E}^{\lambda}(\xi, \xi)=1-\lambda(\xi) \quad(\xi \in T) \quad \text { and } \quad p_{E}^{\lambda}(\xi, v)=\lambda(\xi) p_{E}(\xi, v) \quad(v \neq \xi) .
$$

Since this transformation of processes is invertible, a process with transition probability $\tilde{p}_{E}$ such that $\tilde{p}_{E}(\xi, \xi)>0$ for some $\xi$ can be induced in this way from one with $p_{E}(u, u)=0$ for all $u$.

If we indicate associated objects by $\mu^{\lambda}, c^{\lambda}, Q^{\lambda}$ etc., then

$$
\mu^{\lambda}(\xi)=\frac{1}{Z} \frac{\mu(\xi)}{\lambda(\xi)} \text { where } Z=\sum_{\xi \in T} \frac{\mu(\xi)}{\lambda(\xi)}
$$

and, on writing $m^{\lambda}(u)=\mu^{\lambda}\left(\pi_{T}(u)\right)$,

$$
m^{\lambda}(u) p_{E}^{\lambda}(u, v)=Z^{-1} m(u) p_{E}(u, v) \quad(v \neq u),
$$

as is easily verified. From this relation it immediately follows that if the original walk is symmetric, so is this walk and vice versa; $c=0$ iff $c^{\lambda}=0 ; c^{*}=0$ iff $\left(c^{\lambda}\right)^{*}=0$.

If the modified walk is symmetric relative to each vertex, so is the original walk since by the assumed symmetry $p_{E}^{\lambda}(u, u)$ must be symmetric relative to each vertex. 
The same is also true for the walk invariant under the rotation through $2 \pi / 3$ about each vertex. In these cases we have $Q^{\lambda}(\theta)=Q(\theta) / Z$ (since $\left.c=0\right)$.

4.6. Symmetry relative to an axis. It may be worth noting that if $d=2$, the mirror symmetry relative to one of two coordinate axes implies that the matrix $Q$ is diagonal. (If $T$ is chosen so as to be mirror symmetric relative to the same axis, the matrices $Q^{\circ}, R, Q_{V}^{\circ}, R_{V}$ are also diagonal.)

To be precise, in the higher dimensions suppose that the graph $(V, E)$ is mirror symmetric relative to the $d-1$ dimensional hyperplane, $M$ say, which is perpendicular to the $d$-th coordinate axis and let $\bar{u}$ denote the mirror image of $u$. It holds that: If a random walk on $V$ is mirror symmetric relative to $M$, namely $p_{E}(u, v)=p_{E}(\bar{u}, \bar{v})$, then $(d, j)$ entries of $Q$ vanish for all $j \neq d$.

Proof. Owing to the assumed mirror symmetry of $X_{n}$,

$$
E_{\xi}\left[f\left(X_{n}\right)\right]=\sum_{u} p_{E}^{n}(\bar{\xi}, \bar{u}) f(u)=\sum_{u} p_{E}^{n}(\bar{\xi}, u) f(\bar{u})=E_{\bar{\xi}}\left[f\left(\bar{X}_{n}\right)\right]
$$

for any bounded function $f$ on $V$. Thus $E_{\xi}\left[X_{n}^{j} X_{n}^{d}\right]=E_{\bar{\xi}}\left[\bar{X}_{n}^{j} \bar{X}_{n}^{d}\right]$, where $u^{j}$ denotes the $j$-component of $u \in \mathbf{R}^{d}$. But this expectation equals $-E_{\bar{\xi}}\left[X_{n}^{j} X_{n}^{d}\right]$ for $j \neq d$ since $\bar{u}^{j}=u^{j}$ and $\bar{u}^{d}=-u^{d}$. Now the proof is finished by applying (2).

\section{EXAmples}

All the examples given below are two dimensional and throughout this section we put

$$
\omega=\frac{1}{2}+i \frac{\sqrt{3}}{2}(i=\sqrt{-1})
$$

and use complex number notation when it is convenient without explicitly mentioning that. In Examples 1 through 4 the transition kernel $p_{E}$ satisfies that

$$
p_{E}(u, v)=p_{E}(v, u) \quad \text { and } \quad p_{E}(v, v)=0
$$

for every $u, v \in V$; this relation implies that the random walk on $V$ is symmetric and

$$
\mu(\xi)=1 / \sharp T \text {. }
$$

Certain cases where (20) is violated are considered in Examples 5 and 6 . The letter $\gamma$ (together with $\alpha, \beta$ ) will be used to denote a positive number (not an element of $\Gamma$ nor Euler's constant) except for a few occasions, which will cause no confusion. The parameters that will define $p_{E}$ in each model will be supposed positive in order to make sure that the walk is irreducible, unless stated otherwise explicitly. We write $\theta_{1}$ and $\theta_{2}$ for the two components of $\theta \in \mathbf{R}^{2}: \theta=\left(\theta_{1}, \theta_{2}\right)$.

1. Triangular lattice (0). This example (with $\sharp T=1$ ) may be seen as a usual random walk on $\mathbf{Z}^{2}$ by a simple linear transformation of the lattice. Let $T=$ $\{0\} ; e_{1}=1, e_{2}=\omega$ and define $p_{E}(0,1)=\alpha, p_{E}(0, \omega)=\beta, p_{E}(\omega, 1)=\gamma$. The numbers $\alpha, \beta, \gamma$ are positive and obey the constraint $\alpha+\beta+\gamma=1 / 2$. We have $h=0,|\operatorname{det} A|=\sqrt{3} / 2, \operatorname{det} Q=\sigma^{4}=\frac{3}{8}-\frac{3}{2}\left(\alpha^{2}+\beta^{2}+\gamma^{2}\right)$, and $Q(\theta)=$ $\left(1-\frac{3}{2}(\beta+\gamma)\right) \theta_{1}^{2}+\frac{3}{2}(\beta+\gamma) \theta_{2}^{2}+\sqrt{3}(\beta-\gamma) \theta_{1} \theta_{2}$.

2. Hexagonal lattice. In this model $T=\{0, i\}, e_{1}=\sqrt{3} \omega=\frac{1}{2}(\sqrt{3}+i 3), e_{2}=\sqrt{3} \omega^{2}$; $p_{E}(0, i)=\alpha, p_{E}\left(i, e_{1}\right)=\beta, p_{E}\left(i, e_{2}\right)=\gamma$ with $\alpha+\beta+\gamma=1 .\left(\omega^{2}=\frac{1}{2}(-1+i \sqrt{3}).\right)$ 
Since $\sharp T=2$ and the process $\xi_{n}$ is deterministic, we have $\mathbf{p} h=-h$, so that $c=\frac{1}{2} h$. We make some computations to see that $h(i)=-h(0)=\frac{1}{2}[\sqrt{3}(\beta-\gamma)+i(1-3 \alpha)]$ and that

$$
\begin{gathered}
Q^{\circ}(\theta)=\frac{3}{4}(\beta+\gamma) \theta_{1}^{2}+\frac{1}{4}(1+3 \alpha) \theta_{2}^{2}+\frac{\sqrt{3}}{2}(\beta-\gamma) \theta_{1} \theta_{2} ; \\
Q(\theta)=\frac{3}{4}\left(\left[\beta+\gamma-(\beta-\gamma)^{2}\right] \theta_{1}^{2}+3\left(\alpha-\alpha^{2}\right) \theta_{2}^{2}+2 \sqrt{3}(\beta-\gamma) \alpha \theta_{1} \theta_{2}\right) .
\end{gathered}
$$

We also have $|\operatorname{det} A|=3 \sqrt{3} / 2, m(\xi)=1 / 2$ and $\operatorname{det} Q=\frac{27}{4} \alpha \beta \gamma$, and, using these,

$$
Q^{-1}[c(i)-c(0)]=\frac{1}{9 \alpha \beta \gamma}\left(\begin{array}{c}
\sqrt{3}(\beta-\gamma) \alpha \\
2 \beta \gamma-\alpha(\beta+\gamma)
\end{array}\right) .
$$

(Here a matrix acts on a complex number $w=s+i t$ by regarding $w$ as a column vector with entries $s$ and $t$.)

3. In this class of examples $\sharp T=3$ and $p_{T}(\xi, \xi)=0$ for all $\xi \in T$ (in addition to (20) $)$. In such a case $p_{T}(\xi, \eta)=1 / 2$ if $\xi \neq \eta$, for $p_{T}$ is a symmetric stochastic matrix with zero diagonal elements. We see that $\mathbf{p}=\Pi_{0}-\frac{1}{2} \Pi_{1}$, where $\Pi_{0}$ and $\Pi_{1}$ denote the projection operators on the one dimensional space spanned by the eigenfunction 1 (well expressed as $1 \otimes \mu$ ) and on its orthogonal complement, respectively. Thus we have $\mathbf{p} h=-\frac{1}{2} h$, and, recalling $R(\theta)=2\left\langle\mu,\left(h^{*} \cdot \theta\right)(c \cdot \theta)\right\rangle$,

$$
c=\frac{2}{3} h \quad \text { and } \quad R(\theta)=\sum_{\xi}(c(\xi) \cdot \theta)^{2} .
$$

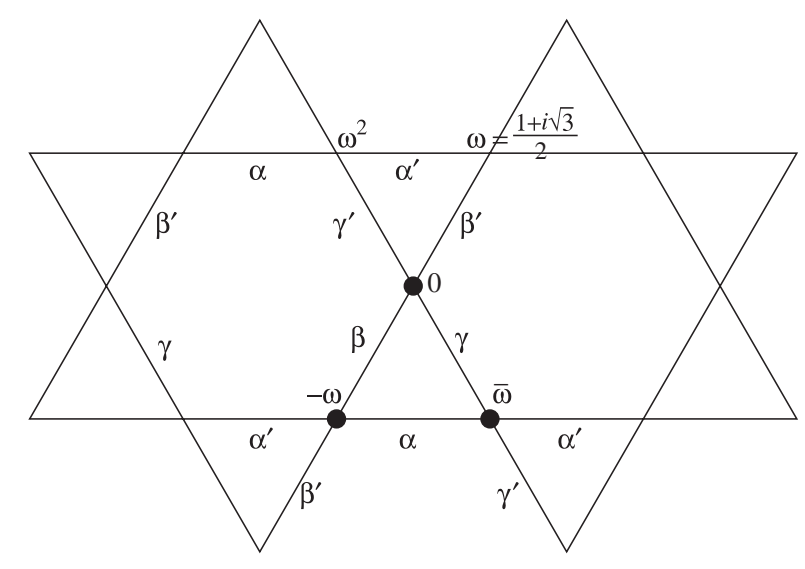

3.1. Kagome lattice I. $T=\{0, \bar{\omega},-\omega\}, e_{1}=2 \omega=1+i \sqrt{3}, e_{2}=2 \omega^{2} .\left(\bar{\omega}=-\omega^{2}.\right)$

$$
\begin{gathered}
p_{E}(-\omega, \bar{\omega})=\alpha, p_{E}\left(\omega, \omega^{2}\right)=\alpha^{\prime} ; \\
p_{E}(0,-\omega)=\beta, p_{E}(0, \omega)=\beta^{\prime} ; p_{E}(0, \bar{\omega})=\gamma, p_{E}\left(0, \omega^{2}\right)=\gamma^{\prime},
\end{gathered}
$$

where $\alpha, \beta$, etc. are positive numbers obeying the constraints $\alpha^{\prime}+\beta+\beta^{\prime}=1$ etc., which may be rewritten as

$$
\alpha^{\prime}=\beta+\beta^{\prime}=\gamma+\gamma^{\prime}=\frac{1}{2} .
$$

We see $Q^{\circ}(\theta)=\frac{1}{2}|\theta|^{2}$ and, setting $r=\alpha-\alpha^{\prime}, s=\beta-\beta^{\prime}$ and $t=\gamma-\gamma^{\prime}$,

$$
h(0)=-s \omega+t \bar{\omega} ; h(\bar{\omega})=-r-t \bar{\omega} ; h(-\omega)=r+s \omega .
$$


Consider the special case $\beta=\gamma$ (implying $r=s=t$ ) and call the common number $p$ :

$$
p:=\beta=\gamma .
$$

Then owing to (21) it is straightforward to see that

$$
g=-i / \sqrt{3}, c(\xi)=(1-4 p)(\xi-g) \quad(\xi \in T) .
$$

The walk is invariant under the rotation through $2 \pi / 3$ about $-i / \sqrt{3}$, so that by 4.3 $Q$ is isotropic. We can now advance the conclusion that $|\operatorname{det} A|=2 \sqrt{3}$,

$$
Q(\theta)=4 p(1-2 p)|\theta|^{2} \text { and } c(\xi)-c(\eta)=(1-4 p)(\xi-\eta) .
$$

Consider another special case when the walk is mirror symmetric relative to the real axis, which means that $\alpha^{\prime}, \beta=\gamma^{\prime}$ and $\gamma=\beta^{\prime}$, so that $r=0, s=-t=\beta-$ $\gamma=2 \beta-\frac{1}{2}$ and $1 / 4$. We see that $c(0)=-\frac{1}{3}(4 \beta-1), c(\bar{\omega})=\frac{1}{3}(4 \beta-1) \bar{\omega}, c(-\omega)=$ $\frac{1}{3}(4 \beta-1) \omega$ and $Q(\theta)=\frac{1}{3}\left(1+4 \beta-8 \beta^{2}\right)|\theta|^{2} .(0<\beta<1 / 2$. $)$

3.2. Triangular lattice I. $T=\{0, \bar{\omega},-\omega\}, e_{1}=1+\omega, e_{2}=1+\bar{\omega}$. We name $\xi_{0}=0, \xi_{1}=\bar{\omega}, \xi_{2}=-\omega$ and $e_{0}=\sqrt{3}$ to facilitate the descriptions. Set for $j=0,1,2$

$$
p_{E}\left(\xi_{j}, \xi_{j+1}\right)=p_{0}^{j+2}, p_{E}\left(\omega^{2} \xi_{j}, \omega^{2} \xi_{j+1}\right)=p_{1}^{j+2}, p_{E}\left(\omega^{4} \xi_{j}, \omega^{4} \xi_{j+1}\right)=p_{2}^{j+2},
$$

where the addition in the super- or subscripts is taken modulo 3 and $p_{k}^{j}$ may be an arbitrary positive number obeying the constraints $p_{0}^{k}+p_{1}^{k}+p_{2}^{k}+p_{0}^{j}+p_{1}^{j}+p_{2}^{j}=1$ $(k \neq j)$, or equivalently,

$$
p_{0}^{j}+p_{1}^{j}+p_{2}^{j}=\frac{1}{2} \quad(j=0,1,2) .
$$
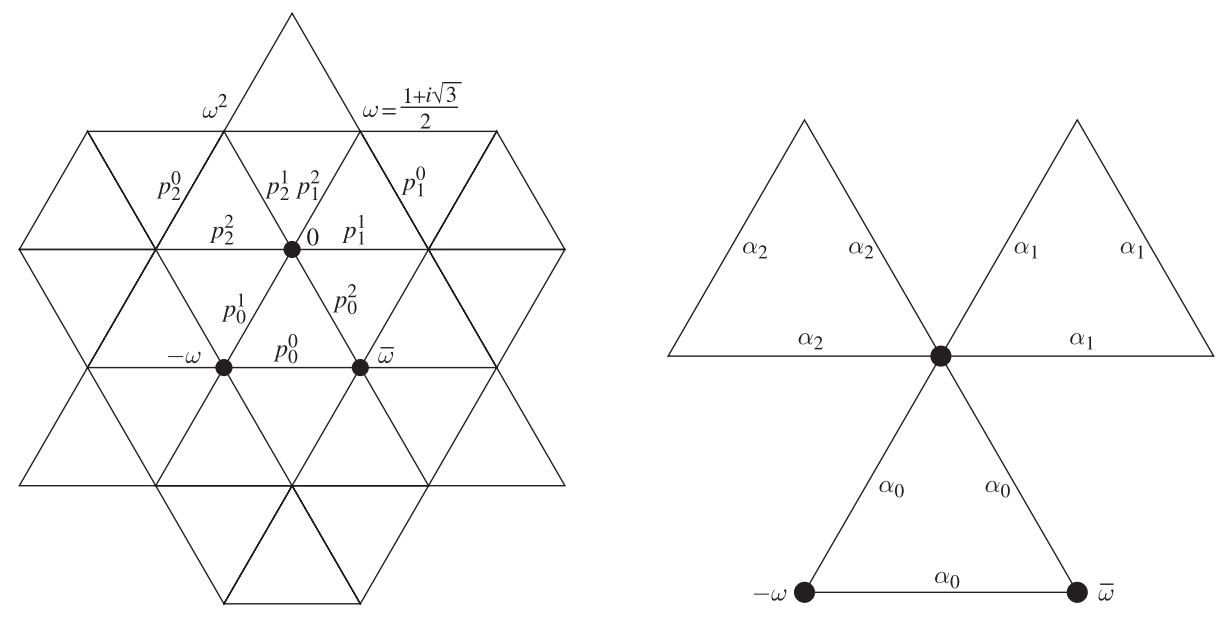

Setting $s_{k}=p_{1+k}^{2+k}-p_{0+k}^{1+k}$ and $t_{k}=p_{2+k}^{1+k}-p_{0+k}^{2+k}$, we have $c\left(\xi_{k}\right)=s_{k}-t_{k}+\frac{i}{\sqrt{3}}\left(s_{k}+\right.$ $\left.t_{k}\right)$.

We specialize the model by equating $p_{j}^{0}, p_{j}^{1}$ and $p_{j}^{2}$ for each $j=0,1,2$ and name them $\alpha_{j}$ :

$$
\alpha_{j}:=p_{j}^{0}=p_{j}^{1}=p_{j}^{2} \quad(j=0,1,2)
$$


Then, on employing (21), $c\left(\xi_{k}\right)=\left(\alpha_{1+k}-\alpha_{2+k}\right)+\frac{i}{\sqrt{3}}\left(\alpha_{1+k}+\alpha_{2+k}-2 \alpha_{k}\right)(k=0,1,2)$ and $Q^{\circ}(\theta)=\frac{1}{2}|\theta|^{2}$. The process is invariant under the rotation through $2 \pi / 3$ about $-i / \sqrt{3}$. Recalling the condition $\alpha_{0}+\alpha_{1}+\alpha_{2}=1 / 2$ we deduce that

$$
\sigma^{2}=\frac{3}{4}\left(1-4\left(\alpha_{0}^{2}+\alpha_{1}^{2}+\alpha_{2}^{2}\right)\right) \text { and } Q(\theta)=\sigma^{2}|\theta|^{2} .
$$

We also have $|\operatorname{det} A|=\frac{3}{2} \sqrt{3}$ and that for $j=0,1,2$,

$$
Q^{-1}\left[c\left(\xi_{j}\right)-c\left(\xi_{j+1}\right)\right]=\frac{1}{\sigma^{2}}\left(\begin{array}{c}
1 / 2-3 \alpha_{j-1} \\
\sqrt{3}\left(\alpha_{j+1}-\alpha_{j}\right)
\end{array}\right) .
$$

Finally we remark that in this model several parameters can be set equal to zero (e.g. we may set $p_{0}^{0}=p_{0}^{1}=p_{0}^{2}=0$ so that there is no edge in $T$ ).

4. Here we give an example with $\sharp T=4, s=2$ and $\tau=1$. Let $T=\{ \pm r, \pm i r\}$; $e_{1}=1, e_{2}=i$ where $r$ may be an arbitrary positive constant (with appropriate interpretation when $r=\frac{1}{2} m$ for some non-negative integer). We suppose, in addition to (20), the walk to be symmetric relative to each coordinate axis, so that it is determined by

$$
p_{E}(\xi, i \xi)=\alpha \text { for } \xi \in T \text { and } p_{E}(r, 1-r)=p_{E}(i r, i(1-r))=\beta,
$$

where $\alpha, \beta$ are positive constants that satisfy $2 \alpha+\beta=1$. Simple computations give

$$
\begin{gathered}
h(\xi)=\left[\beta r^{-1}-(1+\beta)\right] \xi(\xi \in T), \mathbf{p} h=-\beta h, c(\xi)=(1+\beta)^{-1} h ; \\
Q(\theta)=\frac{\beta(1-\beta)}{2(1+\beta)}|\theta|^{2} ; Q^{-1}[c(\xi)-c(\eta)]=\frac{2[\beta-(1+\beta) r]}{\beta(1-\beta) r}(\xi-\eta)(\xi, \eta \in T) .
\end{gathered}
$$

Also, $|\operatorname{det} A|=1$. It may be noted that the second order term vanishes if and only if $r=\beta /(1+\beta)$, which is seen from (3) but not obvious from (15).

5. Here we consider the walks that are symmetric relative to each vertex (or invariant under the rotation through $2 \pi / 3$ for the third one) but do not satisfy the condition (20). By 4.4.1 of Section 4 we have $U=c=c^{*}=0$. We recall that the condition $p_{E}(u, u)=0$ for all $u \in V$, which the succeeding models will satisfy, is not an essential restriction in view of $\mathbf{4 . 5}$.

5.1. Kagome lattice II. Let $(V, E)$ be the Kagome lattice as in Example 3.1. Thus $T=\left\{0, \bar{\omega},-\omega^{2}\right\}, e_{1}=2 \omega, e_{2}=2 \omega^{2}$. We suppose that the walk is symmetric about the origin:

$$
p_{E}(u, v)=p_{E}(-u,-v)
$$

and $p_{E}(u, u)=0$. Since in this lattice $2 \eta \in \Gamma$ for every $\eta \in T$, the condition (22) is equivalent to symmetry of the walk relative to each vertex (see 4.4.2 of Section 4). The transition probability $p_{E}$ is determined by positive numbers $\alpha, \beta, \gamma, \alpha_{-}, \beta_{-}, \gamma_{-}$ as follows:

$$
p_{E}(\bar{\omega},-\omega)=\alpha, p_{E}(-\omega, 0)=\beta, p_{E}(0, \bar{\omega})=\gamma,
$$

and to the respective edges of opposite direction are assigned positive numbers $\alpha_{-}, \beta_{-}$, and $\gamma_{-}$, which obey the constraint

$$
\alpha_{-}+\beta=\beta_{-}+\gamma=\gamma_{-}+\alpha=\frac{1}{2}
$$


and are arbitrary otherwise. One easily finds that

$$
\mu(0)=\frac{1-4 \alpha \alpha_{-}}{Z}, \mu(\bar{\omega})=\frac{1-4 \beta \beta_{-}}{Z}, \mu(-\omega)=\frac{1-4 \gamma \gamma_{-}}{Z},
$$

where $Z=3-2(\alpha+\beta+\gamma)+4(\alpha \beta+\beta \gamma+\gamma \alpha)$. By somewhat tedious computation we obtain

$Q(\theta)=\left[1-\frac{3}{2} \mu(0)\right] \theta_{1}^{2}+\frac{3}{2} \mu(0) \theta_{2}^{2}+Z^{-1}[(1-2 \alpha)(2 \beta-\alpha-\gamma)+4 \alpha \beta(\gamma-\alpha)] \theta_{1} \theta_{2}$.

We may take $\alpha_{-}=\beta_{-}=\gamma_{-}=0$, for which $\tau=s=3, \mu$ is uniform and $Q(\theta)=\frac{1}{2}|\theta|^{2}$. (Similar remarks may be applied to the following two examples.)

Finally observe that the walk $X_{n}$ is symmetric relative to $m$ if $\alpha \beta \gamma=\alpha_{-} \beta_{-} \gamma_{-}$, as is seen from the remark given at the end of $\mathbf{4 . 3 . 2}$ (or by direct computation).

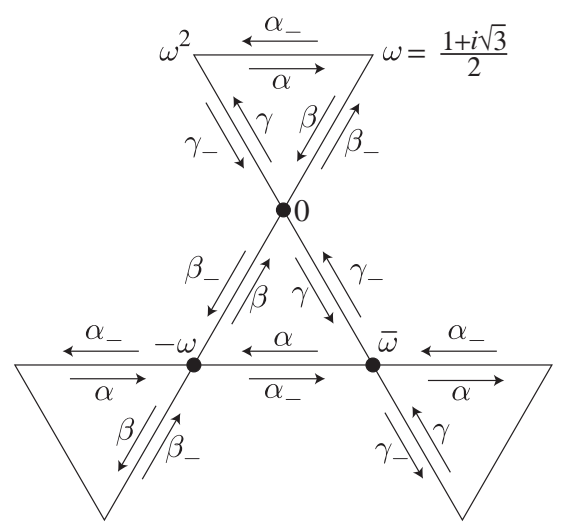

5.2. Triangular lattice II. Consider the graph of Example 3.2, namely $T=$ $\{0, \bar{\omega},-\omega\}, e_{1}=1+\omega, e_{2}=1+\bar{\omega}$. This graph is invariant under the rotation through $2 \pi / 3$ as well as $\pi$.

For this graph every walk that is symmetric relative to each vertex is invariant under shift by $\xi$ for every $\xi \in T$ (for the proof use the invariance under shift by $2 \xi$ besides by $e_{1}$ and $e_{2}$ ), so that it is reduced to a walk on the triangular lattice given in 1.

Any walk that is invariant under the rotation through $2 \pi / 3$ about each vertex is determined by a set of non-negative numbers $\alpha_{ \pm}^{j}$ satisfying $\alpha_{-}^{j}+\alpha_{+}^{j+1}=1 / 3$ $(j=0,1,2)$ as follows. On using the notation of 3.2 ,

$$
p_{0}^{j}=p_{1}^{j}=p_{2}^{j}=\alpha_{+}^{j}, \quad j=0,1,2,
$$

and to the respective edges of opposite direction are assigned the numbers $\alpha_{-}^{j}$. (Notice that in 3.2 the same probability is assigned to both directions of each edge.) By quite simple computation (without computing $\mu$ ) we obtain $Q(\theta)=$ $\frac{1}{2} E_{\mu}\left|Y_{1}\right|^{2}|\theta|^{2}=\frac{1}{2}|\theta|^{2}$.

5.3. Checked square lattice. $e_{1}=1+i, e_{2}=-1+i$ and $T=\left\{0,1, \frac{1}{2} e_{1}\right\}$. We suppose that the walk is symmetric relative to each vertex and $p_{E}(u, u)=0$. Like the Kagome lattice we have $2 \eta \in \Gamma$ for each $\eta \in T$, so that it suffices to check 

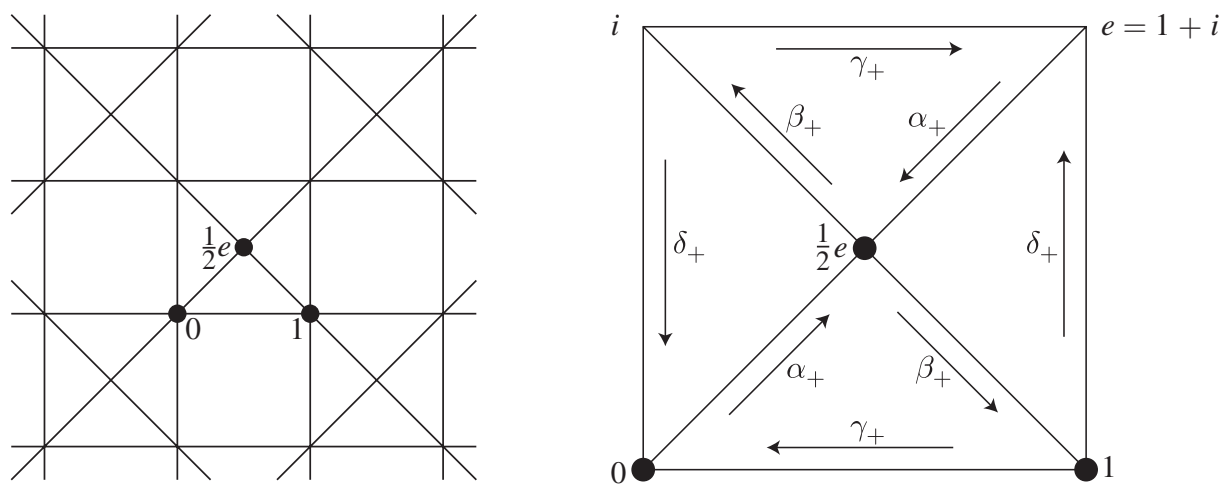

symmetry relative to the origin. The transition probability $p_{E}$ is determined by positive numbers $\alpha_{ \pm}, \beta_{ \pm}, \gamma_{ \pm}$, and $\delta_{ \pm}$as follows:

$$
p_{E}\left(0, \frac{1}{2} e_{1}\right)=\alpha_{+}, p_{E}\left(\frac{1}{2} e_{1}, 1\right)=\beta_{+}, p_{E}(1,0)=\gamma_{+}, p_{E}(i, 0)=\delta_{+} ;
$$

and to the respective edges of opposite direction are assigned the numbers $\alpha_{-},-, \gamma_{-}$ and $\delta_{-}$(omitted in the figure), which are arbitrary except for the constraint

$$
\alpha_{-}+\beta_{+}=\delta_{-}+\gamma_{-}+\alpha_{+}=\delta_{+}+\gamma_{+}+\beta_{-}=\frac{1}{2} .
$$

This together with the symmetry (22) as well as the periodicity determines $p_{E}(u, v)$. It follows that $p_{T}(\xi, \xi)=0$ for $\xi \in T$ and

$$
p_{T}\left(0, \frac{1}{2} e_{1}\right)=2 \alpha_{+}, p_{T}\left(1, \frac{1}{2} e_{1}\right)=2 \beta_{-}, p_{T}\left(\frac{1}{2} e_{1}, 1\right)=2 \beta_{+} ;
$$

the other entries of the matrix $p_{T}$ are determined by the fact that it is stochastic (e.g. $\left.p_{T}(0,1)=1-2 \alpha_{+}\right)$.

By elementary computations, $|\operatorname{det} A|=2$,

$$
\mu(0)=\frac{1-4 \beta_{+} \beta_{-}}{2 Z}, \mu(1)=\frac{1-4 \alpha_{+} \alpha_{-}}{2 Z}, \mu\left(\frac{1}{2} e_{1}\right)=\frac{\alpha_{+}+\beta_{-}-2 \alpha_{+} \beta_{-}}{Z}
$$

( $Z$ is the normalizing constant) and, on using the identity $\mu(0) \alpha_{+}+\mu(1) \beta_{-}=$ $\frac{1}{2} \mu\left(\frac{1}{2} e_{1}\right)$,

$$
\begin{aligned}
Q(\theta)= & \frac{1}{2} \mu\left(\frac{1}{2} e_{1}\right)|\theta|^{2}+[\mu(0)-\mu(1)] \theta_{1} \theta_{2} \\
& +2\left[\mu(0) \gamma_{-}+\mu(1) \gamma_{+}\right] \theta_{1}^{2}+2\left[\mu(0) \delta_{-}+\mu(1) \delta_{+}\right] \theta_{2}^{2} .
\end{aligned}
$$

If the mirror symmetry relative to the diagonal line $x_{1}=x_{2}$ is imposed, which amounts to supposing that $\gamma_{+}=\delta_{+}$and $\gamma_{-}=\delta_{-}$, then

$$
Q(\theta)=\mu(0)\left[\frac{\theta_{1}+\theta_{2}}{\sqrt{2}}\right]^{2}+\mu(1)\left[\frac{\theta_{1}-\theta_{2}}{\sqrt{2}}\right]^{2} .
$$

The function $L(\eta)$ appearing in Corollary 1.1 is identified as follows. Observe that the sum defining $L$ involves only the component $\mu(1)$ among $\mu\left(\pi_{T}(v)\right)$ 's. Then, using $c=c *=0$ and $\sum L(\eta)=\mathrm{v}=\sqrt{2}$, we find that $L(0)=4 \sqrt{2} \gamma_{+}$and $L\left(\frac{1}{2} e\right)=$ $2 \sqrt{2} \beta_{-}$.

In another special case when $\alpha_{+}=\beta_{+}$and $\alpha_{-}=\beta_{-}$(implying that $\mu$ is uniform on $T$ ) the formula (23) reduces to $Q(\theta)=\frac{2}{3}\left[\left(\frac{1}{4}+\gamma_{+}+\gamma_{-}\right) \theta_{1}^{2}+\left(\frac{3}{4}-\gamma_{+}-\gamma_{-}\right) \theta_{2}^{2}\right]$. Here we have three parameters $\alpha_{+}, \gamma_{ \pm}$, which are chosen independently as far as they satisfy the inequalities $0<\alpha_{+}<\frac{1}{2}, 0<\gamma_{+}<\alpha_{+}, 0<\gamma_{-}<\frac{1}{2}-\alpha_{+}$. 
6. In this class of examples each transition law is made up of triangular units of gyration, on each of which the walk gyrates either clockwise or counterclockwise with $p_{E}(u, v)=0$ or $1 / 2$ for every $u, v$. All of them satisfy that $Q^{\circ}(\theta)=\frac{1}{2}|\theta|^{2}$ and that $\mu=1 / 3$, hence $p_{E}^{*}(v, u)=p_{E}(u, v)$, and are invariant under the rotation through $2 \pi / 3$ about $-i / \sqrt{3}$, so that $Q$ is isotropic (see 4.3). The results obtained would provide critical materials for pondering the question of what kind of configuration of gyration units may correspond to more diffusive walks and which correspond to less diffusive ones.

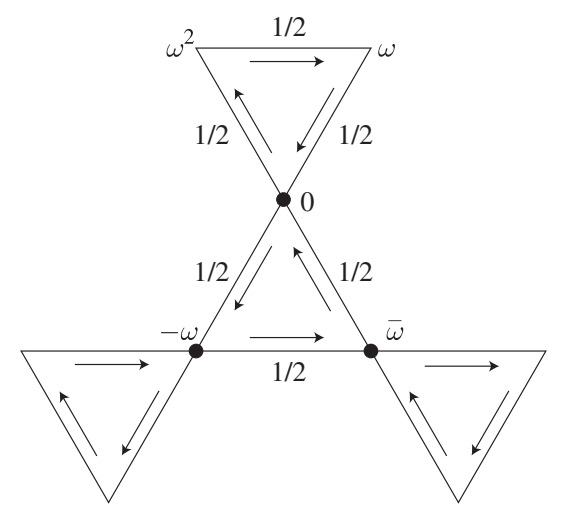

6.1. Kagome lattice III. Consider the graph of Example 3.1 again: $T=\{0, \bar{\omega},-\omega\}$, $e_{1}=2 \omega=1+i \sqrt{3}, e_{2}=2 \omega^{2}$. We set

$$
p_{E}(0,-\omega)=p_{E}(-\omega, \bar{\omega})=p_{E}(\bar{\omega}, 0)=p_{E}\left(0, \omega^{2}\right)=p_{E}\left(\omega^{2}, \omega\right)=p_{E}(\omega, 0)=1 / 2 .
$$

For the other edges the probabilities are uniquely determined by the periodicity, in particular to the (oriented) edges appearing in the list of $p_{E}$ above but of the opposite direction is necessarily assigned zero probability. Notice that the direction of gyration alternates and that the walk is mirror symmetric relative to the real axis. We see that $p_{T}(\xi, \eta)=1 / 2$ if $\xi \neq \eta$, so that $c=\frac{2}{3} h$ (see Example 3 ). Hence $c(0)=-\frac{1}{3}, c(-\omega)=\frac{1}{3} \bar{\omega}, c(\bar{\omega})=\frac{1}{3} \omega$, and by symmetry $c^{*}=-c$. Now

$$
R(\theta)=-\frac{1}{6}|\theta|^{2}, Q(\theta)=\left(\frac{1}{2}+\frac{1}{6}\right)|\theta|^{2} .
$$

Compare this walk with another one, a special case of Example 5.1, which is similar to it except that the walk gyrates clockwise on all unit triangles; for the latter we have $Q(\theta)=\frac{1}{2}|\theta|^{2}$.

6.2. Triangular lattice III. (i) The graph is the same as that of Example 3.2: $T=$ $\{0, \bar{\omega},-\omega\}, e_{1}=1+\omega, e_{2}=1+\bar{\omega}$. As in Example 6.1 we set $p_{E}(\xi, \eta)=0$ if $\xi, \eta \in T$ and

$$
p_{E}(0, \omega)=p_{E}(\omega, 1)=p_{E}(1,0)=p_{E}\left(0, \omega^{2}\right)=p_{E}\left(\omega^{2},-1\right)=p_{E}(-1,0)=1 / 2 .
$$

This walk is mirror symmetric relative to the imaginary axis. (See the first figure on the next page.) The process $\xi_{n}$ is not cyclic $(\tau=1)$ but the walk $X_{n}$ is periodic of period $s=3$ with $V_{0}=T+\left\{x_{1} 3+x_{2} 3 \omega:\left(x_{1}, x_{2}\right) \in \mathbf{Z}^{2}\right\}, V_{1}=V_{0}+e_{1}, V_{1}=V_{0}+e_{2}$. While $c=\frac{2}{3} h(\neq 0)$ as in Example 6.1, we have here $h^{*}=0$. It is readily seen that $Q(\theta)=\frac{1}{2} E_{\mu}\left|Y_{1}\right|^{2}|\theta|^{2}=\frac{1}{2}|\theta|^{2}$. 


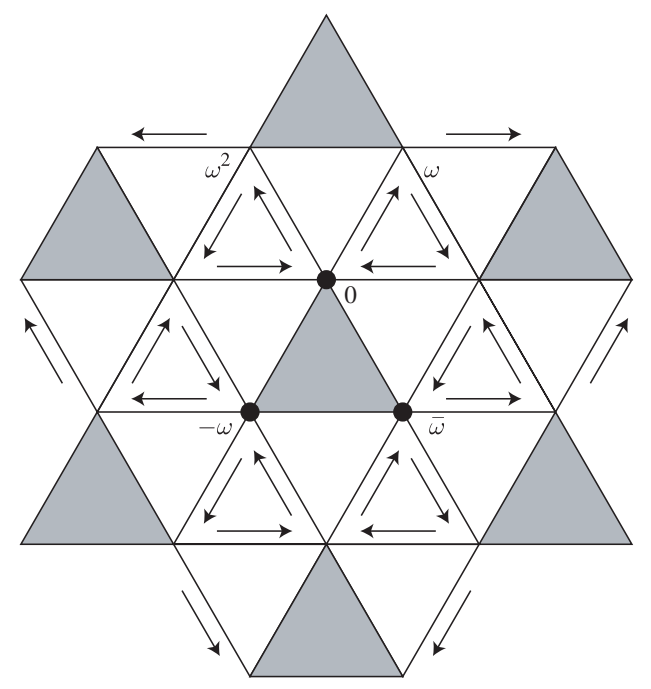

(ii) The walk here is the same as the preceding one except that the direction of the move on the triangle $\triangle 01 \omega$ and all its shifts by $\gamma \in \Gamma$ is reversed so that the walk gyrates counter-clockwise on every unit triangle (see the figure below). Let $\iota$ denote the permutation on $T$ given by $\iota(0)=-\omega, \iota(-\omega)=\bar{\omega}$ and $\iota(\bar{\omega})=0$. Then $\mathbf{p} f=f \circ \iota$, which together with the identity $h+h \circ \iota+h \circ \iota^{2}=0$ leads first to $c=\lim _{r \uparrow 1} \sum_{n=0}^{\infty}(r \mathbf{p})^{n} h=-\frac{1}{3}\left(h \circ \iota+2 h \circ \iota^{2}\right)$, and then to

$$
c(0)=\frac{1}{6}\left(\omega+\omega^{2}\right), c(-\omega)=\frac{1}{6}(-1-\omega), c(\bar{\omega})=\frac{1}{6}(1+\bar{\omega}) .
$$

By symmetry this yields that $c^{*}=c$. Finally by the invariance under the rotation we readily find that $R(\theta)=\frac{1}{8}|\theta|^{2}$ and $Q(\theta)=\left(\frac{1}{2}-\frac{1}{8}\right)|\theta|^{2}$. (Compare $Q$ of (i) and (ii).)

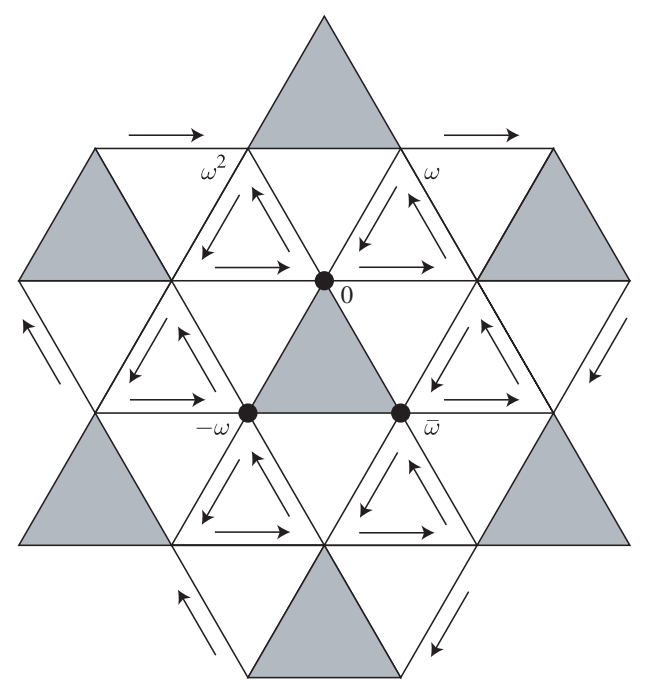


In both examples 6.1 and 6.2 advanced above the diffusivity is smaller for the walks with one and the same direction of gyration than those with the mixed ones. Without knowing exact values this can be deduced by considering the imbedded process $\tilde{X}_{n}$ mentioned at the end of Section 1 and observing two facts concerning it: firstly $P\left[\tilde{X}_{1}=\tilde{X}_{0}\right]=1 / 4$ in all cases and secondly the initial site, say $\xi^{\circ}$, and its six 'neighbors', which are at some equal distance from $\xi^{\circ}$, exhaust the range of $\tilde{X}_{1}$ for the walks gyrating in one direction only, whereas this is not the case for the other walks.

\section{The Walk on a half space (Proof of Theorem 1.4)}

In this section we prove Theorem 1.4. For simplicity we consider the case $d=2$ only. Assume the conditions (a) and (b) as well as the moment condition $E_{\mu}\left[\left|X_{1}\right|^{5}\right]<\infty$. For $u \in \mathbf{R}^{2}$ we denote $u=\left(u_{1}, u_{2}\right)$. We may suppose that $M=\left\{u: u_{2}=0\right\}$ and $e=(0,1)$ so that $V^{+}$is the intersection of $V$ and the upper half plane. Put $q_{e}=e \cdot Q e$.

Observing

$$
\|v-\bar{u}\|^{2}=\|\bar{v}-u\|^{2}=\|v-u\|^{2}+\sigma^{2} 4 u_{2} v_{2} / q_{e}
$$

we see

$$
-\log \frac{\|v-u\|}{\|\bar{v}-u\|}=\frac{\sigma^{2} 2 u_{2} v_{2}}{q_{e}\|v-u\|^{2}}\left[1+O\left(\frac{u_{2} v_{2}}{\|v-u\|^{2}}\right)\right]
$$

$\left(\right.$ as $\left.u_{2} v_{2} /|v-u|^{2} \rightarrow 0\right)$. Also we have

$$
(v-u) \cdot K_{u, v}-(\bar{v}-u) \cdot K_{u, \bar{v}}=\left[2 v_{2} c(\xi) \cdot e+2 u_{2} c^{*}(\eta) \cdot e\right] / q_{e},
$$

where $\xi=\pi_{T}(u), \eta=\pi_{T}(v)$ and, owing to the mirror symmetry, $\mid U(v-u)-$ $U(\bar{v}-u)\left|\leq C u_{2} v_{2}\right| v-u \mid$ where $C$ is a constant. From these, together with the basic relation $G^{+}(u, v)=G(u, v)-G(u, \bar{v})$, it is readily seen that the contribution to $G^{+}(u, v) / m(v)|\operatorname{det} A|$ of the first three terms in the expansion given in Theorem 1.2 can be written as

$$
\frac{2}{\pi q_{e}} \frac{u_{2} v_{2}+v_{2}(c(\xi) \cdot e)+u_{2}\left(c^{*}(\eta) \cdot e\right)}{\|v-u\|^{2}}+\frac{u_{2} v_{2}}{|v-u|^{2}} O\left(\frac{\left|v_{1}-u_{1}\right|+u_{2} v_{2}}{|v-u|^{2}}\right) .
$$

Since $G^{+}>0$ and the remainder term is $O\left(|v-u|^{-2}\right)$, we see first that $u_{2}+c(\xi) \cdot e \geq 0$ on $V^{+}$and then, owing to the strong maximum principle, that $u_{2}+c(\xi) \cdot e>0$ on $V^{+}$. Similarly $u_{2}+c^{*}(\xi) \cdot e>0$ on $V^{+}$.

We must identify the contribution of $r(u, v)$, the remainder term in the expansion of $G$ :

$$
r(u, v)-r(u, \bar{v})=\frac{2}{\pi q_{e}} \frac{(c(\xi) \cdot e)\left(c^{*}(\eta) \cdot e\right)}{\|u-v\|^{2}}+\text { a negligible term }
$$

where the negligible term is understood at most of the order of the error (i.e. the second) term in (25). For the proof of (26) we apply the following lemma.

Lemma 6.1. Set for $\xi, \eta \in T$ and $\theta \in \mathbf{R}^{2}$

$$
\begin{gathered}
Q_{\xi}(\theta)=E_{\xi}\left[\left(Y_{1} \cdot \theta\right)^{2}+2\left(Y_{1} \cdot \theta\right) c\left(\xi_{1}\right) \cdot \theta\right], \\
Q_{\xi}^{*}(\theta)=E_{\mu}\left[\left(Y_{1} \cdot \theta\right)^{2}-2 c^{*}\left(\xi_{0}\right) \cdot \theta\left(Y_{1} \cdot \theta\right) \mid \xi_{1}=\xi\right],
\end{gathered}
$$




$$
\begin{gathered}
C_{\xi, \eta}(\theta)=\left[(1-\mathbf{p})^{-1}(Q(\theta)-Q .(\theta))(\xi)+\left(1-\mathbf{p}^{*}\right)^{-1}\left(Q(\theta)-Q_{.}^{*}(\theta)\right)(\eta)\right], \\
B(\theta)=(2 / 3) E_{\mu}\left(\theta \cdot \tilde{Y}_{1}\right)^{3}-2 E_{\mu}\left[\left(\theta \cdot \tilde{Y}_{1}\right)^{2}\left(c^{*}-c\right)\left(\xi_{0}\right) \cdot \theta\right] .
\end{gathered}
$$

Then, as $|w| \rightarrow \infty$,

$$
\begin{aligned}
r(u, v)= & \int_{D} \frac{2(c(\xi) \cdot \theta)\left(c^{*}(\eta) \cdot \theta\right)}{(2 \pi)^{2} Q(\theta)} e^{-i w \cdot \theta} d \theta+\int_{D} \frac{C_{\xi, \eta}(\theta)}{(2 \pi)^{2} Q(\theta)} e^{-i w \cdot \theta} d \theta \\
& +\int_{D} \frac{\left[c(\xi)-c^{*}(\eta)\right] \cdot \theta B(\theta)}{(2 \pi)^{2} Q^{2}(\theta)} e^{-i w \cdot \theta} d \theta+\frac{\left\{w^{4}\right\}}{\|w\|^{6}}+O\left(\frac{1}{|w|^{3}}\right)
\end{aligned}
$$

where $w=v-u, \xi=\pi_{T}(u), \eta=\pi_{T}(v), D$ is a neighborhood of 0 and the coefficients of $\left\{w^{4}\right\}$ are independent of $(\xi, \eta)$.

Proof. See Remark 9 of 12 as well as the proof of Theorem 1.1 given in Section 2.

Proof of Theorem 1.4. From the mirror symmetry we infer the following relations:

i) $B\left(0, \theta_{2}\right)=0$.

ii) $C_{\xi, \eta}\left(0, \theta_{2}\right)-C_{\xi, \bar{\eta}}\left(0, \theta_{2}\right)=0$.

Indeed i) is verified as in 4.6. To show ii), put $f(\xi)=\left(Q-Q_{\xi}^{*}\right)(e)$. Then we observe that $f(\xi)=f(\bar{\xi})$, implying $\left(1-\mathbf{p}^{*}\right)^{-1} f(\eta)=\left(1-p^{*}\right)^{-1} f(\bar{\eta})$. Hence we have ii).

By i) together with (24) the contribution to $r(u, v)-r(u, \bar{v})$ of the third integral in (27) is dominated by a constant multiple of

$$
\sum_{1 \leq k \leq 3} \frac{\left|w_{2}\right|^{k}\left|w_{1}\right|^{4-k}}{|w|^{6}}+\frac{u_{2} v_{2}}{|w|^{4}}=O\left(\frac{\left.u_{2} v_{2}+\left|w_{1}\right|\left|w_{2}\right|\right)}{|w|^{4}}\right)
$$

since the corresponding integrand does not include the term $\theta_{1}^{4} / Q^{2}(\theta)$ nor $\theta_{2}^{4} / Q^{2}(\theta)$. Similarly the second integral is negligible owing to ii) above. By using (24) again it is easy to dispose of the term $\left\{w^{4}\right\} /\|w\|^{6}$.

Finally, we identify the contribution of the first integral with the right side of (26) by employing the identity $c^{*}(\eta) \cdot \theta-c^{*}(\bar{\eta}) \cdot \theta=2\left(c^{*}(\eta) \cdot e\right) \theta_{2}$ together with the following formula: if $Q(\theta)=q_{1} \theta_{1}^{2}+q_{2} \theta_{2}^{2}$, then

$$
\frac{1}{(2 \pi)^{2}} \int_{\mathbf{R}^{2}} \frac{\theta_{2}^{2}}{Q(\theta)} e^{-i w \cdot \theta} d \theta=\frac{\sigma^{2}}{2 \pi q_{2}} \frac{q_{1}^{-1} w_{1}^{2}-q_{2}^{-1} w_{2}^{2}}{\|w\|^{4}}=\frac{1}{2 \pi q_{2}\|w\|^{2}}-\frac{\sigma^{2} w_{2}^{2}}{\pi q_{2}^{2}\|w\|^{4}},
$$

where the Fourier transform is understood in the sense of Schwartz distribution in $\mathbf{R}^{2} \backslash\{0\}$. The proof of Theorem 1.4 is complete.

\section{REFERENCES}

[1] Babillot, M (1988) Théorie du renouvellement pour des chaînes semi-markoviennes transientes, Ann. Inst. H. Poincaré (4) 24, 507-569. MR978023 (90h:60082)

[2] Cinlar, M (1972) Markov additive processes I, II, Z. Wahr.verw. Beb. 24, 85-93, 95-121. MR0329047 (48:7389)

[3] Givarc'H, Y (1984) Application d'un théoreme limite local à la transience et à la récurrence des marches de Markov, Lecture Notes, n. 1096, 301-332, Springer-Verlag.

[4] Keilson, J and Wishart, D M G (1964) A central limit theorem for processes defined on a finite Markov chain, Proc. Cambridge Philos. Soc. 60, 547-567. MR0169271 (29:6523)

[5] Krámli, A And SzÁsz, D (1983) Random walks with internal degrees of freedom, Z. Wahr. verw. Gebiete, 63, 85-95. MR699788 (85f:60098) 
[6] Kotani, M, Shirai, T and Sunada, T (1998) Asymptotic behavior of the transition probability of a random walk on an infinite graph, J. Func. Anal. 159, 664-689. MR.1658100 (2000c:60058)

[7] Kotani, M And Sunada, T (2000) A central limit theorem for the simple random walk on a crystal lattice, Proceedings of the Second ISAAC Congress, Vol. 1 (Fukuoka, 1999), 1-6, Int. Soc. Anal. Appl. Comput., 7, Kluwer Acad. Publ., Dordrecht. MR1940777 (2003h:60036)

[8] Kotani, M And Sunada, T (2000) Albanese maps and off diagonal long time asymptotics for the heat kernel, Comm. Math. Phys. 209, 633-670. MR1743611 (2001h:58036)

[9] Takenami, T (2004) Local limit theorem for random walk in periodic environment, Osaka Jour. Math. 39, 867-895. MR.1951520 (2004a:60093)

[10] Spitzer, F (1964) Principles of Random Walks, Van Nostrand, Princeton. MR0171290 $(30: 1521)$

[11] Uchiyama, K (1998) Green's functions for random walks on $\mathbf{Z}^{d}$, Proc. London Math. Soc. 77, 215-240. MR1625467 (99f:60132)

[12] Uchiyama, K (2007) Asymptotic estimates of Green's functions and transition probabilities for Markov additive processes, Elec. J. Probab. 12 (2007), 138-180. MR2299915

Department of Mathematics, Tokyo Institute of Technology, Oh-okayama, Meguro TOKYO, 152-8551 JAPAN

E-mail address: uchiyama@math.titech.ac.jp

Department of Mathematics, Tokyo Institute of Technology, Oh-okayama, Meguro TOKYO, 152-8551 JAPAN 